\title{
YOUNG STELLAR OBJECTS IN THE GOULD BELT
}

\author{
Michael M. Dunham ${ }^{1}$, Lori E. Allen ${ }^{2}$, Neal J. Evans II $^{3}$, Hannah Broekhoven-Fiene ${ }^{4}$, Lucas A. Cieza ${ }^{5,6}$, \\ James Di Francesco $^{7}$, Robert A. Gutermuth ${ }^{8}$, Paul M. Harvey ${ }^{3}$, Jennifer Hatchell ${ }^{9}$, Amanda Heiderman ${ }^{10,11}$, \\ Tracy L. Huard ${ }^{12,13}$, Doug Johnstone ${ }^{7,14}$, Jason M. Kirk ${ }^{15}$, Brenda C. Matthews ${ }^{7}$, Jennifer F. Miller ${ }^{16}$, \\ Dawn E. Peterson ${ }^{17}$, AND KaISA E. Young ${ }^{18}$ \\ ${ }^{1}$ Harvard-Smithsonian Center for Astrophysics, 60 Garden Street, MS 78, Cambridge, MA 02138, USA; mdunham@cfa.harvard.edu \\ ${ }^{2}$ National Optical Astronomy Observatories, Tucson, AZ, USA \\ ${ }^{3}$ Department of Astronomy, The University of Texas at Austin, 2515 Speedway, Stop C1400, Austin, TX 78712-1205, USA \\ ${ }^{4}$ Department of Physics \& Astronomy, University of Victoria, Victoria, BC, V8W 3P6, Canada \\ ${ }^{5}$ Núcleo de Astronomía de la Facultad de Ingeniería, Universidad Diego Portales, Av. Ejército 441, Santiago, Chile \\ ${ }^{6}$ Millenium Nucleus "Protoplanetary Disks in ALMA Early Science," Chile \\ ${ }^{7}$ National Research Council of Canada, Herzberg Astronomy \& Astrophysics Programs, 5071 West Saanich Road, Victoria, BC, V9E 2E7, Canada \\ ${ }^{8}$ Department of Astronomy, University of Massachusetts, Amherst, MA 01003, USA \\ ${ }^{9}$ Physics and Astronomy, University of Exeter, Stocker Road, Exeter EX4 4QL, UK \\ ${ }^{10}$ Department of Astronomy, University of Virginia, P.O. Box 400325, Charlottesville, VA 22904, USA \\ ${ }^{11}$ National Radio Astronomy Observatory, 520 Edgemont Road, Charlottesville, VA 22903, USA \\ ${ }_{12}$ Department of Astronomy, University of Maryland, College Park, MD 20742, USA \\ ${ }^{13}$ Columbia Astrophysics Laboratory, Columbia University, New York, NY 10027, USA \\ ${ }^{14}$ Department of Physics and Astronomy, University of Victoria, Victoria, BC V8P 1A1, Canada \\ ${ }^{15}$ Jeremiah Horrocks Institute, University of Central Lancashire, Preston, PR1 2HE, UK \\ ${ }^{16}$ Gemini Observatory, 670 N. A'ohoku Place, Hilo, HI 96720, USA \\ ${ }^{17}$ Space Science Institute, 4750 Walnut Street, Suite 205, Boulder, CO 80301, USA \\ ${ }^{18}$ Department of Physical Sciences, Nicholls State University, P.O. Box 2022, Thibodaux, LA 70310, USA \\ Received 2015 May 20; accepted 2015 August 12; published 2015 September 9
}

\begin{abstract}
We present the full catalog of Young Stellar Objects (YSOs) identified in the 18 molecular clouds surveyed by the Spitzer Space Telescope "cores to disks" (c2d) and "Gould Belt" (GB) Legacy surveys. Using standard techniques developed by the c2d project, we identify 3239 candidate YSOs in the 18 clouds, 2966 of which survive visual inspection and form our final catalog of YSOs in the GB. We compile extinction corrected spectral energy distributions for all 2966 YSOs and calculate and tabulate the infrared spectral index, bolometric luminosity, and bolometric temperature for each object. We find that $326(11 \%), 210(7 \%), 1248(42 \%)$, and $1182(40 \%)$ are classified as Class $0+$ I, Flat-spectrum, Class II, and Class III, respectively, and show that the Class III sample suffers from an overall contamination rate by background Asymptotic Giant Branch stars between 25\% and 90\%. Adopting standard assumptions, we derive durations of $0.40-0.78 \mathrm{Myr}$ for Class $0+\mathrm{I}$ YSOs and $0.26-0.50 \mathrm{Myr}$ for Flat-spectrum YSOs, where the ranges encompass uncertainties in the adopted assumptions. Including information from (sub)millimeter wavelengths, one-third of the Class $0+$ I sample is classified as Class 0, leading to durations of $0.13-0.26 \mathrm{Myr}$ (Class 0) and $0.27-0.52 \mathrm{Myr}$ (Class I). We revisit infrared color-color diagrams used in the literature to classify YSOs and propose minor revisions to classification boundaries in these diagrams. Finally, we show that the bolometric temperature is a poor discriminator between Class II and Class III YSOs.
\end{abstract}

Key words: infrared: stars - ISM: clouds - stars: formation - stars: low-mass

Supporting material: machine-readable tables

\section{INTRODUCTION}

Most of the current star formation within $500 \mathrm{pc}$ of the Sun occurs in the Gould Belt (GB), a ring of nearby O-type stars inclined approximately $20^{\circ}$ with respect to the Galactic Plane (Herschel 1847; Gould 1879). The Sun is located interior to the GB, which has a radius of about 350 pc (e.g., Clube 1967; Stothers \& Frogel 1974; Comeron et al. 1992; de Zeeuw et al. 1999; Pöppel 2001). All of the nearby, well-studied giant molecular clouds (e.g., Ophiuchus, Taurus, Perseus, Serpens, Orion, etc.) are located within this ring (see Figure 1 of WardThompson et al. 2007, for a schematic diagram of the GB in galactic coordinates).

Due to the high visual extinction toward giant molecular clouds, a complete census of star formation in these clouds requires infrared observations. The Infrared Astronomical Satellite (IRAS) provided the first such unbiased survey of the GB (and the rest of the sky), but was limited in both sensitivity and spatial resolution. For young stellar objects (YSOs) still in the embedded phases of evolution, IRAS was only sensitive to objects with luminosities above $0.1(d / 140 \mathrm{pc})^{2} L_{\odot}$ (Myers et al. 1987). For more evolved YSOs, IRAS was even less sensitive. The spatial resolution provided by IRAS ranged from 0.5 at $12 \mu \mathrm{m}(0.02-0.07 \mathrm{pc}$ at distances of $150-500 \mathrm{AU})$ to $2^{\prime}$ at $100 \mu \mathrm{m}(0.09-0.29 \mathrm{pc}$ at distances of $150-500 \mathrm{AU})$, insufficient to resolve individual stars forming in clustered regions of molecular clouds. Ground-based near-infrared surveys (e.g., the 2MASS; Skrutskie et al. 2006) provide much higher spatial resolution but are unable to detect the most deeply embedded, heavily extincted, and/or lowest luminosity YSOs. Ground-based mid-infrared studies are generally limited by the atmosphere to only the brightest and most luminous objects. Finally, selected regions of the GB were mapped in the mid-infrared by the Infrared Space Observatory (ISO). While ISO surveys identified many new deeply embedded and low 

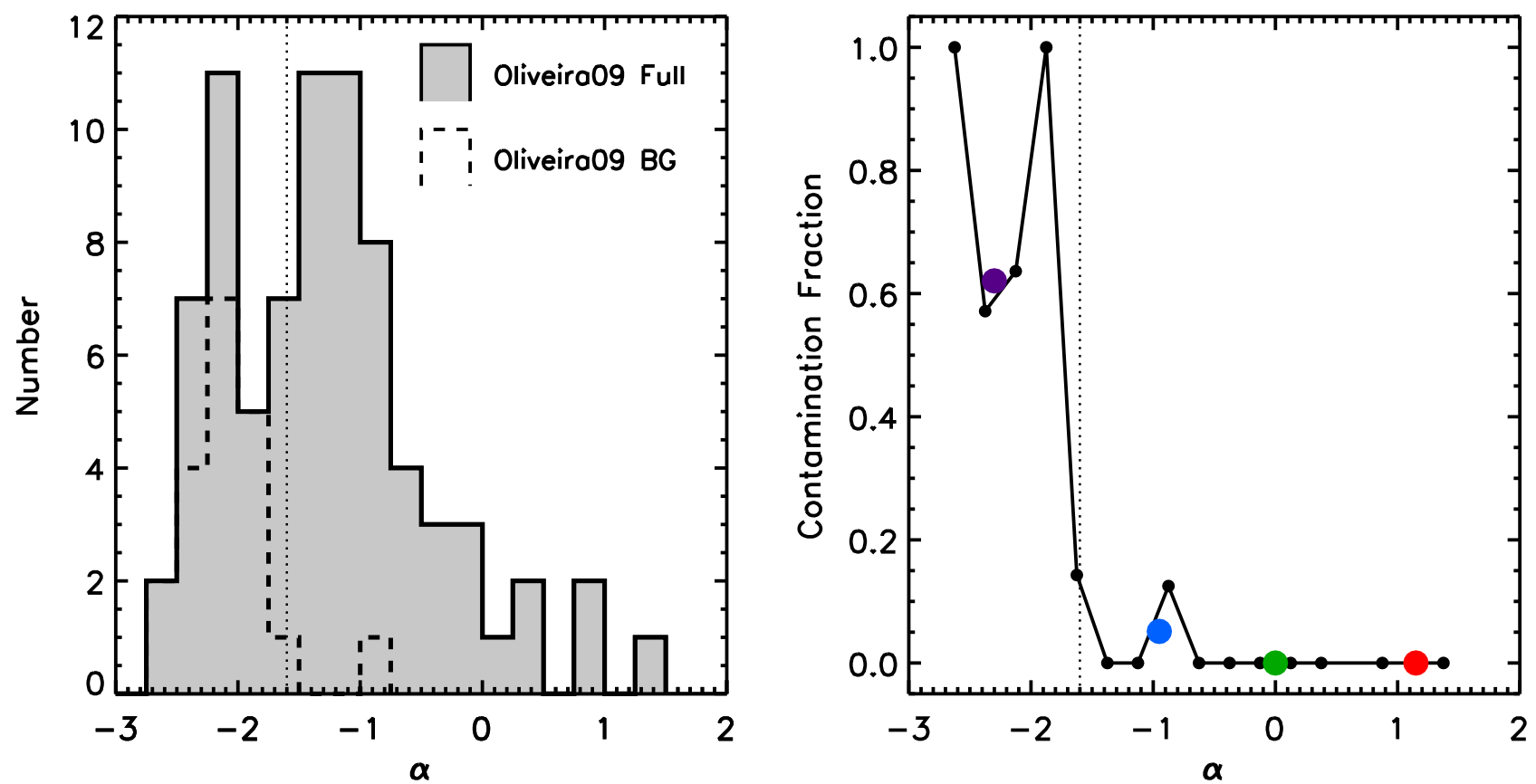

Figure 1. Left: histograms of the infrared spectral index ( $\alpha$, uncorrected for extinction) for the YSOs observed by Oliveira et al. (2009). The shaded histogram shows the full sample of 78 objects whereas the dashed histogram shows the 20 objects identified as background AGB stars by Oliveira et al. Right: the black symbols and line plot the contamination fraction in each $\alpha$ bin, where this fraction is defined as the number of background AGB stars in this bin divided by the total number of objects in this bin, using the results from Oliveira et al. (2009). The colored symbols plot the contamination fraction for each Class (red for Class $0+\mathrm{I}$, green for flatspectrum, blue for Class II, and purple for Class III). The Class II and Flat-spectrum results are plotted at the mean value of $\alpha$ for each class, whereas the Class III and Class $0+$ I results are plotted at the mean values of the boundaries for each Class and the display ranges of the $x$ axis. The dotted line in each panel marks the Class III boundary $(\alpha<-1.6)$.

luminosity YSOs, they only focused on small portions of molecular clouds (e.g., Bontemps et al. 2001; Persi et al. 2003; Kaas et al. 2004).

The launch of the Spitzer Space Telescope (Werner et al. 2004) in 2003 opened new avenues for infrared surveys of giant molecular clouds. With its combination of high sensitivity and large format detector arrays operating at 3.6-160 $\mu \mathrm{m}$, Spitzer was the first mid-infrared facility capable of mapping entire molecular clouds within reasonable amounts of time. The Spitzer Legacy project "From Molecular Cores to Planet-forming Disks" (Cores to Disks, or c2d) took advantage of these features by mapping seven large, nearby GB molecular clouds and nearly 100 isolated dense cores. An overview of the survey is given by Evans et al. (2003) and a summary of results for the large clouds is given by Evans et al. (2009). The c2d survey identified over 1000 YSOs, many of which were newly identified, and demonstrated Spitzer's ability to detect YSOs that are too deeply embedded, too low luminosity, and/or too low mass to be detected by previous facilities.

The Spitzer GB Legacy survey followed up the c2d survey by mapping an additional $11 \mathrm{~GB}$ clouds using the same observation and data reduction strategies as c2d. Combined, the two surveys targeted nearly every major site of star formation within $500 \mathrm{pc}$ of the Sun. The only two exceptions are the Taurus and Orion Molecular Clouds, both of which are so large they were the subject of their own, dedicated surveys by different teams (Rebull et al. 2010; Megeath et al. 2012). Adding the results of the GB survey to those obtained by $\mathrm{c} 2 \mathrm{~d}$ nearly triples the number of YSOs identified in the GB. A preliminary attempt to combine the results from the c2d, GB, Taurus, and Orion surveys was presented by Dunham et al. (2014), but a full combination, including reconciliation of different methods for observing, data reduction, source extraction and classification, is left for future work that combines the expertise of the respective surveys. Here we only consider the $\mathrm{c} 2 \mathrm{~d}$ and GB surveys.

While the full list of YSOs identified by Spitzer in the c2d and GB surveys has been used in several studies, including those on clustering of young stars (Bressert et al. 2010), star formation rates and thresholds (Heiderman et al. 2010), the luminosities of protostars (Dunham et al. 2013), relationships between gas and star formation (Evans et al. 2014), and the physical nature of empirically defined classes of young stars (Heiderman \& Evans 2015), this list itself has not yet appeared in publication. To rectify this, we present in this paper the full list of YSOs in the combined c2d and GB clouds. For the $\mathrm{c} 2 \mathrm{~d}$ clouds, this list updates and replaces the previous version given by Evans et al. (2009), including an analysis of contamination by background Asymptotic Giant Branch (AGB) stars, updated extinction corrections, and revised spectral energy distributions (SEDs). Although we use this YSO list to investigate classification of young stars and the durations of these classes, the primary purpose of this work is to present a full catalog of Spitzer $\mathrm{c} 2 \mathrm{~d}+\mathrm{GB}$ YSOs. While useful on its own, this YSO list will reach its full potential in combination with results from the far-infrared and submillimeter surveys of the GB by the Herschel Space Observatory and SCUBA-2 on the James Clerk Maxwell Telescope, once they become fully available (WardThompson et al. 2007; André et al. 2010; Harvey et al. 2013). By providing the full Spitzer YSO list, we enable such comparisons.

The organization of this paper is as follows. We describe our method in Section 2, including an overview of the c2d and GB surveys (Section 2.1), the procedure for identifying YSOs 
Table 1

Molecular Clouds Surveyed by the c2d and GB Surveys

\begin{tabular}{|c|c|c|c|c|c|c|}
\hline Cloud & Survey & $\begin{array}{l}\text { Distance } \\
(\mathrm{pc})\end{array}$ & $\begin{array}{c}\text { Distance } \\
\text { Reference }^{\mathrm{a}}\end{array}$ & $\begin{array}{l}\text { Area } \\
\left(\mathrm{pc}^{2}\right)\end{array}$ & $\begin{array}{l}\text { Mass } \\
\left(M_{\odot}\right)\end{array}$ & $\begin{array}{c}\text { Data } \\
\text { Reference(s) }\end{array}$ \\
\hline Aquila & GB & 260 & 1 & 179.0 & $24400 \pm 3000$ & 1 \\
\hline Auriga/CMC & GB & 450 & 2 & 52.4 & $4844 \pm 1152$ & 2 \\
\hline Cepheus & GB & $200-325^{\mathrm{c}}$ & 3 & 38.0 & $2610 \pm 170$ & 3 \\
\hline Chamaeleon I & GB & 150 & 4 & 9.4 & $857 \pm 210$ & $\ldots$ \\
\hline Chamaeleon II & $\mathrm{c} 2 \mathrm{~d}$ & 178 & 5 & 9.2 & $637 \pm 300$ & $4,5,6$ \\
\hline Chamaeleon III & GB & 150 & 4 & 28.0 & $1330 \pm 390$ & $\ldots$ \\
\hline Corona Australis & GB & 130 & 6 & 3.0 & $279 \pm 110$ & 7 \\
\hline IC5146 & GB & 950 & 7 & 149.0 & $8550 \pm 2170$ & 8 \\
\hline Lupus I & $\mathrm{c} 2 \mathrm{~d}$ & 150 & 8 & 8.9 & $513 \pm 310$ & 9,10 \\
\hline Lupus III & $\mathrm{c} 2 \mathrm{~d}$ & 200 & 8 & 15.4 & $912 \pm 320$ & 9,10 \\
\hline Lupus IV & $\mathrm{c} 2 \mathrm{~d}$ & 150 & 8 & 2.5 & $189 \pm 95$ & 9,10 \\
\hline Lupus V & GB & 150 & 8 & 11.7 & $705 \pm 220$ & 11 \\
\hline Lupus VI & GB & 150 & 8 & 6.7 & $455 \pm 140$ & 11 \\
\hline Musca & GB & 160 & 9 & 6.8 & $335 \pm 110$ & $\ldots$ \\
\hline Ophiuchus & $\mathrm{c} 2 \mathrm{~d}$ & 125 & 10 & 29.6 & $3120 \pm 1800$ & 12 \\
\hline Ophiuchus North & GB & 130 & 11 & 7.3 & $621 \pm 17$ & 13 \\
\hline Perseus & $\mathrm{c} 2 \mathrm{~d}$ & 250 & 12 & 73.2 & $6590 \pm 3600$ & 14,15 \\
\hline Serpens & $\mathrm{c} 2 \mathrm{~d}$ & 429 & 13 & 17.0 & $2340 \pm 640$ & $16,17,18$ \\
\hline
\end{tabular}

Notes.

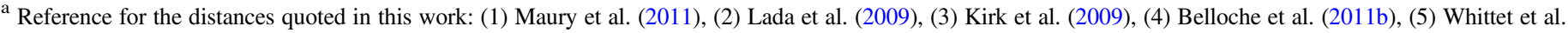

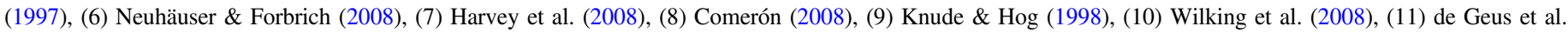
(1989), (12) Enoch et al. (2006), (13) Dzib et al. (2011).

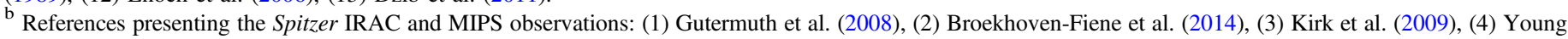

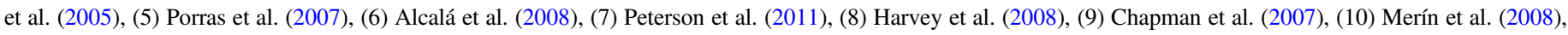

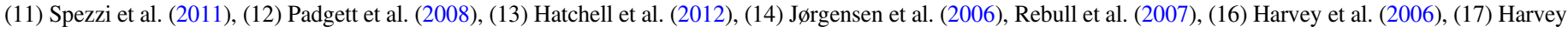
et al. (2007b), (18) Harvey et al. (2007a).

${ }^{c}$ Different regions within Cepheus are located at different distances; see Kirk et al. (2009) for details.

(Section 2.2), residual contamination in our YSO sample by background AGB stars (Section 2.3), and the construction of full SEDs and corrections for extinction (Section 2.4). We present our results in Section 3, including the full list of YSOs (Section 3.1) and a discussion of classification of young stars (Sections 3.2-3.3). We discuss timescales for the classes of young stars in Section 4. Finally, we summarize our results in Section 5 .

\section{METHOD}

A concise summary of these surveys, the adopted procedure for identifying YSOs, and the methods used to construct SEDs and to correct the photometry for foreground extinction are given in Section 2 of Dunham et al. (2013). Here we repeat the key information and refer the reader to Dunham et al. for more details.

\subsection{Overview of the Surveys}

The Spitzer c2d survey (PI: N. J. Evans) imaged seven large, nearby molecular clouds in the GB and approximately 100 isolated dense molecular cores. Evans et al. (2003) review both the primary science objectives of $\mathrm{c} 2 \mathrm{~d}$ and its basic observation plan, and Evans et al. (2009) summarize the results in the large clouds. The Spitzer GB survey (PI: L. E. Allen) is a follow-up program that imaged an additional 11 molecular clouds in the GB, completing most of the remaining clouds in the GB except for the Taurus and Orion molecular clouds (see Section 1). Both surveys imaged their respective targets at $3.6-8.0 \mu \mathrm{m}$ with the Spitzer Infrared Array Camera (IRAC; Fazio et al. 2004), and at 24-160 $\mu \mathrm{m}$ images with the Multiband Imaging
Photometer (MIPS; Rieke et al. 2004). A data pipeline developed by $\mathrm{c} 2 \mathrm{~d}$ was applied to the observations obtained by both programs. This pipeline includes data reduction, source extraction, and band-merging the results from each wavelength to produce final images and source catalogs, and has been described in detail elsewhere (Harvey et al. 2006; Evans et al. 2007).

For each cloud targeted by $\mathrm{c} 2 \mathrm{~d}$ or GB, Table 1 lists the parent survey, the assumed distance and reference for the distance, the total area mapped, the total cloud gas mass, and references to in-depth studies of each cloud based on the $\mathrm{c} 2 \mathrm{~d}$ or GB data, when available. The Spitzer Astronomical Observation Requests (AORs) for each cloud can be found in these references to individual cloud studies; Appendix A lists the AORs for the clouds without published data references. The isolated cores surveyed by $\mathrm{c} 2 \mathrm{~d}$ are not considered here except for a few cases where the cores are actually parts of larger cloud complexes and included in the results for those clouds (namely, Cepheus and Ophiuchus North; Kirk et al. 2009; Hatchell et al. 2012). Note that the distances to these clouds are not all well determined, and some cloud distances are still under debate. One such example is the uncertainty over the distance to Aquila, with two distances (260 and $429 \mathrm{pc}$ ) commonly adopted in the literature (see, e.g., Harvey et al. 2006; Gutermuth et al. 2008; Dzib et al. 2011; Maury et al. 2011, for details). For this reason we do not list formal distance uncertainties and instead refer the reader to the references given in Table 1 for full discussions of the distances to each cloud.

The total cloud areas mapped and total cloud gas masses contained within these areas are taken from Heiderman et al. 
(2010), with the masses determined from extinction maps (see Heiderman et al. for details). For most clouds, the mapped areas were chosen to map the entire cloud above a minimum visual extinction in the range $2<A_{\mathrm{V}}<3$, with the exact value for each cloud depending on the total area that could be mapped within the allocated time. For Serpens and Aquila, only the cloud areas above $A_{\mathrm{V}}=6$ were mapped due to time constraints and difficulty in separating the cloud from the general extinction close to the Galactic midplane.

\subsection{Identification of YSOs}

The data reduction pipeline used by both the $c 2 d$ and GB surveys creates band-merged source catalogs where the sources extracted at each Spitzer wavelength are matched with each other and with the 2MASS catalog, creating a final catalog of $1.25-70 \mu \mathrm{m}$ photometry for each cloud. Candidate YSOs are identified using a method developed by Harvey et al. (2007a) and summarized in all of the publications focused on individual clouds that are listed in Table 1. In this method, the Spitzer SWIRE Legacy survey of the ELAIS N1 extragalactic field (Lonsdale et al. 2003) is processed to simulate the same sensitivity and extinction distribution of the c2d and GB clouds, and then used to locate background galaxies observed through the target clouds in various infrared color-magnitude diagrams. Using these results to define the expected colors and magnitudes of background galaxies, each source extracted in the cloud maps with infrared colors that can not be explained as an extincted background star is assigned an unnormalized "probability" of being a galaxy or YSO based on its position in each color-magnitude diagram, its morphology in the two shortest Spitzer IRAC bands (3.6 and $4.5 \mu \mathrm{m}$ ), and its 24 and $70 \mu \mathrm{m}$ flux densities. Since it is unnormalized, this quantity is not a true probability but a relative parameter expressing the likelihood of being a background galaxy (higher values indicate higher likelihood of being galaxies; see Harvey et al. 2007a, for details). The final boundary between candidate YSO and candidate galaxy in this quantity is set to provide a nearly complete elimination of SWIRE sources, likely at the cost of excluding YSOs with overlapping colors and magnitudes (e.g., Hsieh \& Lai 2013).

One potential drawback to the $c 2 d+G B$ method of identifying YSOs is that it requires detections in all four IRAC bands $(3.6-8.0 \mu \mathrm{m})$ as well as the first MIPS band $(24 \mu \mathrm{m})$. As the $24 \mu \mathrm{m}$ MIPS band has lower resolution and sensitivity, some of the faintest and/or most clustered YSOs may be missed (e.g., Gutermuth et al. 2009, R.A. Gutermuth et al. 2015, in preparation). We note that several alternative classification methods for separating background galaxies and YSOs have been presented in the literature (e.g., Gutermuth et al. 2009; Rebull et al. 2010; Kryukova et al. 2012; Hsieh \& Lai 2013). While they are all based on a similar philosophy of separating sources in various colors and magnitudes, they differ in their detailed implementations of this philosophy. A full comparison of these methods and ultimate consensus on best practices for extracting YSOs from infrared surveys of starforming clouds is left for future work.

Applying these selection criteria results in the identification of 3239 candidate YSOs in the $18 \mathrm{c} 2 \mathrm{~d}$ and GB clouds. We note here that we only consider sources that are located in the overlap regions imaged by both the IRAC and MIPS instruments, as these are the sources that can be most reliably classified using the method summarized above. For technical reasons, the MIPS observations often covered much larger areas (see the references listed in Table 1 for more details on the observations of each individual cloud). Several of the individual cloud studies listed in Table 1 also present candidate YSO lists in the MIPS-only regions using alternative identification and classification techniques, but such sources are not considered here. We visually inspected each of the 3239 candidate YSOs to remove resolved galaxies and image artifacts that are sometimes misidentified (see Evans et al. 2009, for details). Additionally, a few known YSOs that were saturated or undetected at one or more wavelengths were missing from the initial list and were added by hand. In the end, we were left with a final list of 2966 YSOs, and since all have passed visual inspection, we follow Evans et al. (2009) and drop the prefix "candidate" at this point.

Finally, we note that initial results from Herschel surveys of the $\mathrm{c} 2 \mathrm{~d}$ and GB clouds suggest that a modest number of the most deeply embedded protostars are undetected by Spitzer, increasing the number of Class 0 protostars $^{19}$ by $15 \%-30 \%$ (e.g., Harvey et al. 2013; Stutz et al. 2013; Sadavoy et al. 2014). Revisions to the YSO lists presented here will thus be necessary once complete Herschel results are available for each cloud.

\subsection{Background Giant Star Contamination}

Our final sample of YSOs is likely contaminated by background giant stars with infrared excesses. Oliveira et al. (2009) performed optical spectroscopy toward 78 YSOs in Serpens and identified $20(26 \%)$ as background AGB stars. While the overall contamination rate is relatively low, Figure 1 shows that the contamination fraction strongly depends on the infrared spectral index $\alpha .^{20}$ Starting from positive values, as $\alpha$ decreases, the contamination fraction remains close to zero until the Class II/III boundary is reached at $\alpha=-1.6$ (see Section 3.2 for a full definition and discussion of YSO classes), and then steeply rises to $>50 \%$ for more negative values of $\alpha$. On average, $62 \%$ of the Class III sources examined by Oliveira et al. are actually background AGB stars, whereas only $5 \%$ of the Class II sources they examined were found to be contaminants.

Very high rates of AGB star contamination among Class III sources are also found by Romero et al. (2012), also with optical spectra. They found Class III contamination rates of $100 \%$ based on observations of 30 objects in Lupus V and VI. While the fact that they only observed objects that met initial selection criteria for transition disks may bias their results, they observed $45 \%$ and $39 \%$ of the total Lupus V and VI Class III populations, respectively; thus, the true contamination fractions are at least $\sim 40 \%$ in each cloud.

The true contamination fraction is likely a strong function of galactic latitude, and the specific clouds considered above (Serpens, Lupus V, and Lupus VI) all lie within $2^{\circ}-10^{\circ}$ of the galactic plane. Indeed, Romero et al. (2012) found a lower contamination rate of $25 \%$ for Ophiuchus $\left(b=18^{\circ}\right)$, consistent with the Ophiuchus contamination rate of $20 \%$

\footnotetext{
${ }^{19}$ Class 0 protostars are the youngest class of YSOs; see Section 3.1 for the formal definition of this class of objects.

${ }^{20}$ The quantity $\alpha$ is defined as the slope of the infrared SED in $\log \left(\lambda S_{\lambda}\right)$ versus $\log (\lambda)$ and is used to classify YSOs, as discussed in detail in Section 3.1 and Section 3.2. While $\alpha$ calculated from extinction corrected photometry is used in later sections, here we use the values calculated from the observed photometry for consistency with the previous studies to which we compare.
} 


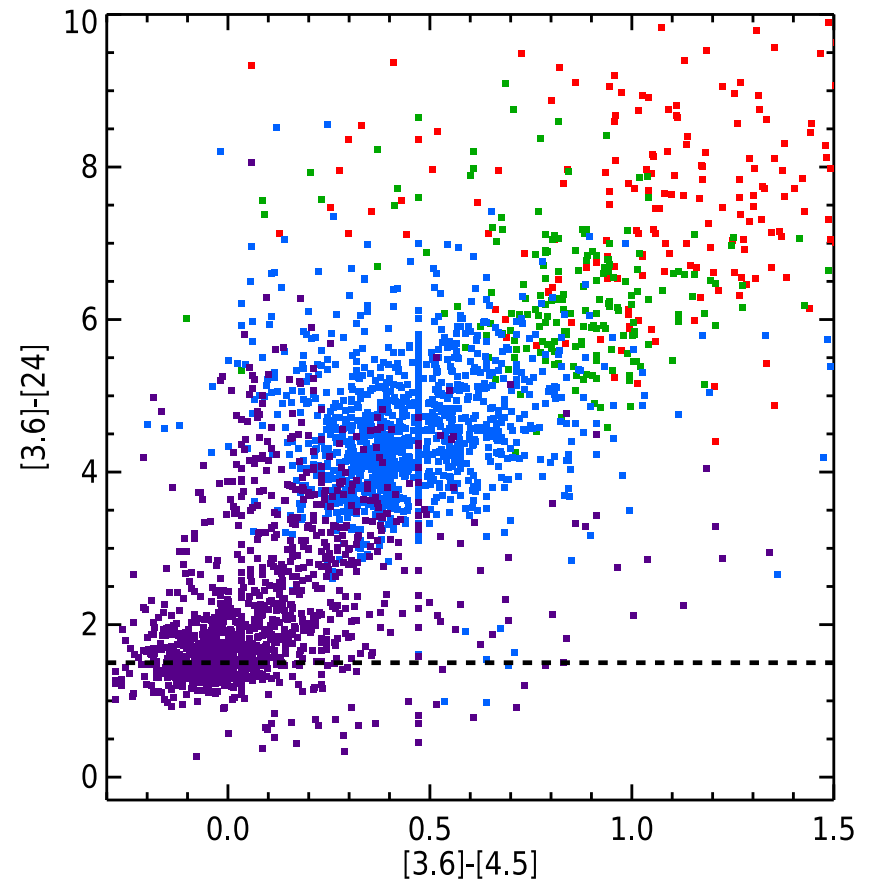

Figure 2. [3.6]-[24] vs. [3.6]-[4.5] for all 2966 objects in our final YSO sample, using the observed photometry. The color of each symbol shows its spectral class determined using the extinction corrected infrared spectral index (see Sections 3.1 and 3.2 for details on classification), with red indicating Class $0+\mathrm{I}$, green indicating Flat-spectrum, blue indicating Class II, and purple indicating Class III. The horizontal dashed line indicates the boundary of [3.6]$[24]=1.5$ used to identify highly likely AGB stars contaminating our YSO sample. The vertical line of sources at [3.6]-[4.5] 0.5 is a rounding artifact that is discussed in detail in Appendix B.

measured by Cieza et al. (2010) using similar methods. To further investigate the contamination rate in our full sample of YSOs, Figure 2 plots a Spitzer color-color diagram consisting of [3.6]-[24] versus [3.6]-[4.5], using the observed photometry. Inspection of Figure 2 shows that the Class III YSO population can be divided into a population of objects clustered at lower (bluer) values of both colors, and a second, more distributed population extending to higher (redder) colors. Using optical spectra of candidate transition disks, Cieza et al. (2010, 2012) and Romero et al. (2012) found that background AGB stars are predominately found at low values of [3.6]-[24], suggesting that the clustered population in Figure 2 may be dominated by AGB stars whereas the more distributed population may be dominated by YSOs. Indeed, the above studies found that all of their objects with [3.6]-[24] $\leqslant 1.5$ were in fact AGB stars, with decreasing contamination rates at higher colors. We find that 298 of the objects in our final YSO sample have [3.6]-[24] $\leqslant 1.5$, all but three of which are classified as Class III objects. This represents $25 \%$ of the Class III YSO sample and $10 \%$ of the total YSO sample. We consider these objects as likely AGB stars and mark them as such throughout the remainder of this paper. However, we do not remove them from the sample since the studies cited above only targeted a subset of objects that were identified as transition disk candidates and thus there is no guarantee that their finding of $100 \%$ contamination at the lowest values of [3.6]-[24] applies to our full sample. Confirmation of the status of these objects requires either spectroscopic or proper motion studies. Such work should be considered as a high priority for

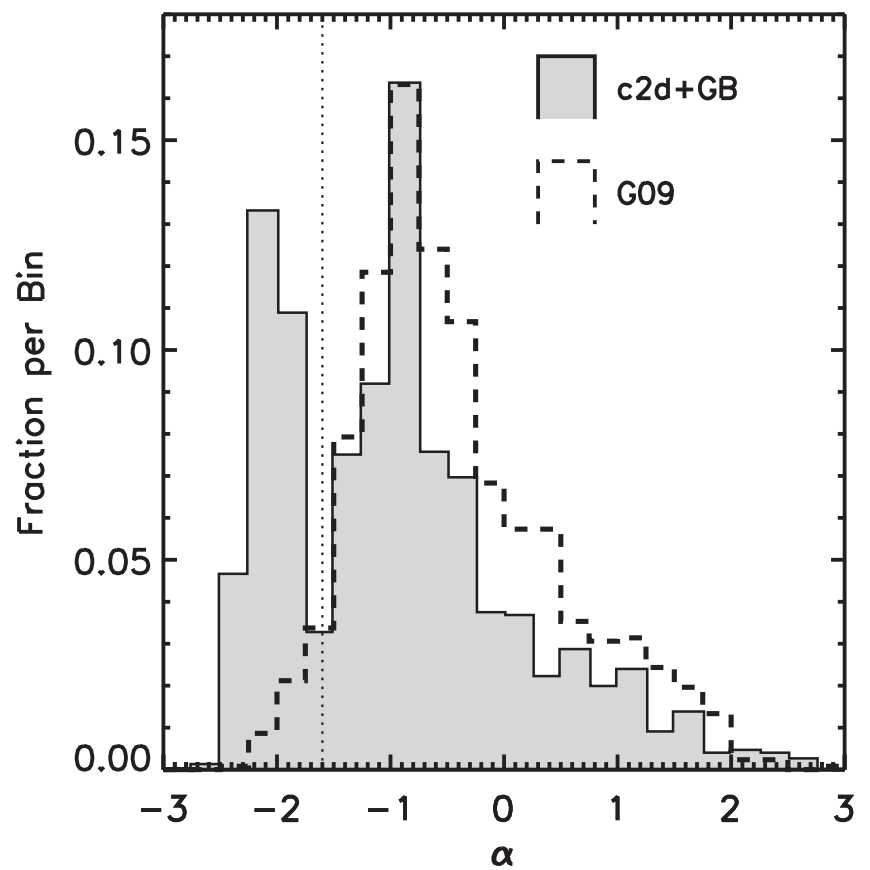

Figure 3. Histogram showing the distribution of infrared spectral index for the YSOs identified in the $\mathrm{c} 2 \mathrm{~d}+\mathrm{GB}$ clouds (this work, shaded histogram) and by Gutermuth et al. (2009) in a survey of young stellar clusters (dashed histogram). The dotted line marks the Class III boundary $(\alpha<-1.6)$.

future investigations seeking to refine the YSO sample presented here.

The $25 \%$ Class III contamination by AGB stars found above is almost certainly a lower limit to the full number, since some contaminating AGB stars are found to have [3.6]-[24] $>1.5$ (Cieza et al. 2010, 2012; Romero et al. 2012). However, these studies target samples that are too small and too biased to determine contamination rates as a function of [3.6]-[24] color. To obtain an upper limit to the possible contamination fraction, Figure 3 compares the distributions of $\alpha$ for our YSOs with those identified in young stellar clusters by Gutermuth et al. (2009), using a selection method that is also based on infrared colors and magnitudes but is different in its details (see Gutermuth et al. for details). The $\mathrm{c} 2 \mathrm{~d}+\mathrm{GB}$ sample contains a much higher fraction of Class III objects. While a full comparison of different methods for identifying YSOs is beyond the scope of this work, under the assumptions that the Gutermuth et al. method perfectly separates out background AGB stars and our regions contain the same fractions of sources in each Class, $87 \%$ of the Class III objects in the c $2 d+G B$ sample would need to be removed to match the Gutermuth et al. (2009) Class III fraction. We note here that the Gutermuth et al. survey targeted smaller regions centered on dense clusters whereas the $\mathrm{c} 2 \mathrm{~d}$ and GB surveys mapped much larger areas, including those more distant from cluster centers. As the Class II and III YSO populations are generally more distributed than earlier Classes, the intrinsic fraction of Class III sources is likely higher in the c2d and GB surveys than in Gutermuth et al. (2009). For this reason, our above assumption that the surveys contain the same fractions of sources in each Class results in an upper limit to the Class III contamination fraction.

Synthesizing all of the above information, our sample of Class III YSOs suffers from between $25 \%$ and $90 \%$ contamination by AGB stars. These values are lower and 
upper limits, respectively, for the reasons listed above, and the most likely value is somewhere in between. Tighter constraints on the true contamination rate will require large optical spectroscopic campaigns and/or proper motion studies coupled with revisions to the selection criteria used to identify YSOs in infrared surveys. These contamination rates must be kept in mind when considering the results presented here and in other studies based on the $\mathrm{c} 2 \mathrm{~d}+\mathrm{GB}$ YSO list (such as those discussed in Section 1).

\subsection{Constructing SEDs and Correcting for Extinction}

Following the method first summarized by Evans et al. (2009) and updated by Dunham et al. (2013), we compile as complete an SED as possible for each of the 2966 YSOs. We refer to Dunham et al. for a full description of this process, but give a summary here. To construct the SEDs, we included the following.

1. Selected ground-based optical and infrared photometry as compiled by the authors of the individual cloud papers listed in Table 1.

2. Wide-field Infrared Survey Explorer (WISE; Wright et al. 2010) 12 and $22 \mu \mathrm{m}$ photometry from the all-sky catalog. $^{21}$

3. Spitzer $160 \mu \mathrm{m}$ photometry for sources that are detected but not located in saturated or confused regions. Since the c2d pipeline does not extract $160 \mu \mathrm{m}$ sources, we measured flux densities using aperture photometry (using the IDL procedure APER) and standard aperture corrections as given by the MIPS Instrument Handbook. ${ }^{22}$

4. Selected $350 \mu \mathrm{m}$ photometry from a targeted survey of protostars with SHARC-II ${ }^{23}$ (Wu et al. 2007, Suresh et al. 2015).

5. Selected 450 and $850 \mu \mathrm{m}$ photometry from the $\mathrm{SCUBA}^{24}$ Legacy Catalog (Di Francesco et al. 2008).

6. Other submillimeter and millimeter photometry from the literature, where available from large surveys of nearby molecular clouds (Young et al. 2005, 2006; Enoch et al. 2006, 2007; Belloche et al. 2011a, 2011b; Maury et al. 2011).

Regarding the last three items, we have access to complete submillimeter or millimeter surveys for only 6 out of the 18 clouds (Chamaeleon I, Chamaeleon II, Chamaeleon III, Ophiuchus, Perseus, and Serpens), plus a partial survey of Aquila and piecemeal coverage of other clouds from the SCUBA Legacy Catalog (Di Francesco et al. 2008) and our own $350 \mu \mathrm{m}$ observations (Wu et al. 2007, A. Suresh et al. 2015, submitted). We consider a Spitzer source to match a submillimeter or millimeter detection if it is located within one beam of the detection, using the appropriate beam size for each survey. If multiple Spitzer sources match a single submillimeter or millimeter detection, we do not attempt to split the flux density and simply match it to the closest

\footnotetext{
21 Available at http://irsa.ipac.caltech.edu/cgi-bin/Gator/nph-scan?mission = irsa\&submit $=$ Select\&projshort $=$ WISE

${ }^{22}$ Available at http://irsa.ipac.caltech.edu/data/SPITZER/docs/mips/ mipsinstrumenthandbook/

23 The Submillimeter High Angular Resolution Camera II (SHARC-II) was a $350 \mu \mathrm{m}$ bolometer array operated at the Caltech Submillimeter Observatory (Dowell et al. 2003).

24 The Submillimeter Common-User Bolometer Array (SCUBA) was a 450 and $850 \mu \mathrm{m}$ bolometer array operated at the James Clerk Maxwell Telescope.
}

Spitzer source unless the SED clearly indicates a better match is obtained with a different Spitzer source.

Finally, we correct all photometry for foreground extinction following the procedure outlined by Evans et al. (2009). We only wish to correct for the foregound extinction from both the clouds and the material between us and the clouds, and not the local extinction from dense cores surrounding protostars (this extincted emission is reprocessed to longer wavelengths and thus included in our compiled SEDs). Thus, we adopt extinction values as follows.

1. Whenever possible, we adopt extinction values from the literature for Class II and III YSOs (classified via infrared spectral index; see Section 3.2), as determined from optical or near-infrared spectroscopic studies of each cloud that provide reliable spectral type (and thus extinction) measurements. We refer to the data references listed in Table 1 for more details on these studies.

2. For all Class II and III YSOs with no published extinction values, we de-redden to the intrinsic near-infrared colors of an assumed spectral type of K7. This particular spectral type is chosen because it is found to be representative of the YSOs in the c2d clouds (Oliveira et al. 2009, 2010, see also Evans et al. 2009 for details).

3. We calculate the mean extinction toward all Class II YSOs in each cloud, and then adopt that mean value for each of the Class $0+\mathrm{I}$ and Flat-spectrum YSOs in that cloud.

With extinctions assigned as above, we de-redden the SED of each YSO using the Weingartner \& Draine (2001) extinction law for $R_{V}=5.5$. Whereas Evans et al. (2009) adopted a version of the Weingartner \& Draine (2001) $R_{V}=5.5$ extinction law that only included dust absorption, here we update the procedure to correct for both dust absorption and scattering. The choice of this particular extinction law is motivated by results showing that it is appropriate for the dense molecular clouds in which stars form (e.g., Chapman et al. 2009). While we do caution that our approach only provides approximate extinction corrections, especially for the Class $0+$ I and Flat-spectrum sources where the corrections only account for the mean cloud extinction, it is nevertheless the best that can currently be done and is significantly more reliable than ignoring the effects of extinction altogether.

\section{RESULTS}

\subsection{Full List of YSOs}

For each of the 2966 YSOs, Table 2 lists a running index, the cloud in which the YSO is found, the Spitzer source name (which also gives the source coordinates), the visual extinction used for extinction corrections, and the infrared spectral index $(\alpha)$, the bolometric temperature $\left(T_{\mathrm{bol}}\right)$, and the bolometric luminosity $\left(L_{\mathrm{bol}}\right)$. These last three quantities are calculated using both the observed and extinction corrected photometry, and are denoted with primes in the latter case $\left(\alpha^{\prime}, T_{\mathrm{bol}}^{\prime}\right.$, and $\left.L_{\mathrm{bol}}^{\prime}\right)$. The second-to-last column of Table 2 indicates whether or not each object has [3.6]-[24] $\leqslant 1.5$ and is thus likely a background AGB star, as described above in Section 2.3.

The final column of Table 2 indicates whether or not each object is associated with a dense core as traced by submillimeter or millimeter continuum emission at $\lambda \geqslant$ $350 \mu \mathrm{m}$. As a lack of such an association does not necessarily 
Table 2

Properties of the $\mathrm{c} 2 \mathrm{~d}+\mathrm{GB}$ YSOs

\begin{tabular}{|c|c|c|c|c|c|c|c|c|c|c|c|}
\hline \multirow[b]{2}{*}{ Index } & \multirow[b]{2}{*}{ Cloud } & \multirow{2}{*}{$\begin{array}{c}\text { Spitzer } \\
\text { Source Name } \\
(\text { SSTc2d or SSTgb }+)\end{array}$} & \multirow[b]{2}{*}{$\begin{array}{c}A_{\mathrm{V}} \\
(\mathrm{mag})\end{array}$} & \multicolumn{3}{|c|}{ Observed } & \multicolumn{3}{|c|}{ Extinction Corrected } & \multirow[b]{2}{*}{$\begin{array}{l}\text { Likely } \\
\text { AGB? }\end{array}$} & \multirow[b]{2}{*}{ Core? } \\
\hline & & & & $\alpha$ & $\begin{array}{l}T_{\text {bol }} \\
(\mathrm{K})\end{array}$ & $\begin{array}{l}L_{\text {bol }} \\
\left(L_{\odot}\right)\end{array}$ & $\alpha^{\prime}$ & $\begin{array}{l}T_{\text {bol }}^{\prime} \\
(\mathrm{K})\end{array}$ & $\begin{array}{l}L_{\text {bol }}^{\prime} \\
\left(L_{\odot}\right)\end{array}$ & & \\
\hline 1 & Aquila & J180144.8-044941 & 8.2 & -2.16 & 1700 & 3.0 & -2.52 & 2000 & 8.2 & $\mathrm{~N}$ & $\ldots$ \\
\hline 2 & Aquila & J180145.8-045902 & 8.0 & -2.14 & 1700 & 1.4 & -2.56 & 2000 & 3.7 & $\mathrm{~N}$ & $\ldots$ \\
\hline 3 & Aquila & $\mathrm{J} 180154.2-043753$ & 12.4 & -2.57 & 2100 & 0.52 & -3.22 & 2600 & 4.7 & $\mathrm{Y}$ & $\ldots$ \\
\hline 4 & Aquila & $\mathrm{J} 180229.2-051552$ & 8.3 & -2.03 & 1700 & 3.6 & -2.38 & 2000 & 9.8 & $\mathrm{~N}$ & $\ldots$ \\
\hline 5 & Aquila & J180302.0-044233 & 12.4 & -2.45 & 2100 & 0.11 & -3.15 & 2500 & 0.92 & $\mathrm{~N}$ & $\ldots$ \\
\hline 6 & Aquila & J180308.0-043523 & 8.0 & -2.18 & 1700 & 0.24 & -2.52 & 2000 & 0.64 & $\mathrm{Y}$ & $\ldots$ \\
\hline 7 & Aquila & $\mathrm{J} 180313.8-042845$ & 6.9 & -2.22 & 1800 & 0.72 & -2.53 & 2000 & 1.7 & $\mathrm{Y}$ & $\ldots$ \\
\hline 8 & Aquila & J180423.9-042248 & 9.0 & -2.15 & 1700 & 2.2 & -2.44 & 2000 & 6.3 & $\mathrm{Y}$ & $\ldots$ \\
\hline 9 & Aquila & J180435.7-044700 & 8.1 & -2.26 & 1700 & 1.9 & -2.65 & 2000 & 5.3 & $\mathrm{Y}$ & $\ldots$ \\
\hline 10 & Aquila & J180444.5-043706 & 12.4 & 0.53 & 540 & 0.055 & -0.10 & 1000 & 0.11 & $\mathrm{~N}$ & $\ldots$ \\
\hline
\end{tabular}

(This table is available in its entirety in machine-readable form.)

imply that no core is present (see below), to avoid any confusion in the interpretation of this column we list either $\mathrm{Y}$ or no entry rather than Y or N. Dunham et al. (2013) used such associations to define the sample of protostars in the $\mathrm{c} 2 \mathrm{~d}+\mathrm{GB}$ clouds, with the rationale behind this definition being that detections in existing submillimeter and millimeter surveys of star-forming regions trace dense cores but not circumstellar disks due to the relatively low spatial resolutions and mass sensitivities of these surveys (see Dunham et al. 2013, for details). By requiring a dense core but making no assumptions about the infrared colors of protostars, this definition ensures a reliable sample of protostars that includes those viewed edgeon down outflow cavities, but comes at the cost of incompleteness to those protostars that are embedded in lowmass cores or located in clouds with incomplete or no available submillimeter or millimeter continuum surveys (see Dunham et al. 2014, for details). Associations with dense cores and the resulting sample of protostars must be revisited once the James Clerk Maxwell telescope (JCMT) SCUBA-2 survey of the GB is complete, the first results of which are just starting to become available (e.g., Pattle et al. 2015).

The infrared spectral index $\left(\alpha, \alpha^{\prime}\right)$ is defined as the slope of the infrared SED in $\log \left(\lambda S_{\lambda}\right)$ versus $\log (\lambda)$, where $\lambda$ is the wavelength and $S_{\lambda}$ is the flux density at that wavelength:

$$
\alpha=\frac{d \log \left(\lambda S_{\lambda}\right)}{d \log (\lambda)} .
$$

In practice, we calculate $\alpha$ with a linear least squares fit to all available 2MASS and Spitzer photometry between 2 and $24 \mu \mathrm{m}$. The bolometric temperature of a source is defined to be the temperature of a blackbody with the same flux-weighted mean frequency (Myers \& Ladd 1993). It is in essence a protostellar equivalent of stellar effective temperature; it starts at very low values $(\sim 10 \mathrm{~K})$ for cold, starless cores and eventually increases to the effective temperature of the central (proto)star once the core and disk have fully dissipated. $L_{\mathrm{bol}}$, $L_{\mathrm{bol}}^{\prime}, T_{\mathrm{bol}}$, and $T_{\mathrm{bol}}^{\prime}$ are calculated by integrating over the full SEDs:

$$
L_{\mathrm{bol}}=4 \pi d^{2} \int_{0}^{\infty} S_{\nu} d \nu
$$

$$
T_{\text {bol }}=1.25 \times 10^{-11} \frac{\int_{0}^{\infty} \nu S_{\nu} d \nu}{\int_{0}^{\infty} S_{\nu} d \nu} \mathrm{K} .
$$

In practice, we calculate these integrals using the trapezoid rule to integrate over the finitely sampled SEDs.

Figure 4 plots $L_{\text {bol }}^{\prime}$ versus $T_{\text {bol }}^{\prime}$ (a "BLT" diagram; Myers \& Ladd 1993) for all 2966 YSOs identified in the c2d and GB clouds. The luminosities of Class 0 and Class I sources are distributed over greater than 4 orders of magnitude and extend to much lower luminosites than predicted by simple evolutionary models. A full discussion of this "protostellar luminosity problem" can be found in Offner \& McKee (2011), Dunham et al. (2013, 2014), but to summarize, massdependent and/or time variable mass accretion is required to explain the observed luminosities of protostars. Class II and Class III objects exhibit both a very narrow range of bolometric temperatures and a bifurcation in $T_{\text {bol }}^{\prime}$ into two groups. Both effects are artifacts introduced by our extinction corrections and are discussed in Appendix C. To summarize, the first effect is because the bolometric temperature of a Class II or III source depends more on the spectral type of the object than the amount and characteristics of circumstellar material, and for most objects a constant spectral type of K7 is assumed (see above). The second effect (bifurcation) is a consequence of optical data only being available for a subset of our YSOs.

Tables 3 and 4 list, for each YSO, the same running index as in Table 2, followed by flux density and flux density uncertainty pairs for the 2MASS $(1.25,1.65$, and $2.17 \mu \mathrm{m})$ and Spitzer (3.6, 4.5, 5.8, 8.0, 24, and $70 \mu \mathrm{m}$ ) wavelengths. All flux densities and flux density uncertainties are listed in mJy and rounded to two significant digits. Table 3 lists the observed values while Table 4 lists the extinction corrected values. The observed and extinction corrected flux densities that we have compiled at all other wavelengths in order to construct SEDS, as described above, are tabulated in Appendix D.

\subsection{Classification of YSOs}

Over the past few decades a standard model for low-mass star formation has emerged. This model, developed by Adams et al. (1987) and summarized by Shu et al. (1987), resulted from the merger of an empirical classification system based on the infrared spectral indices of YSOs (Lada \& Wilking 1984) 


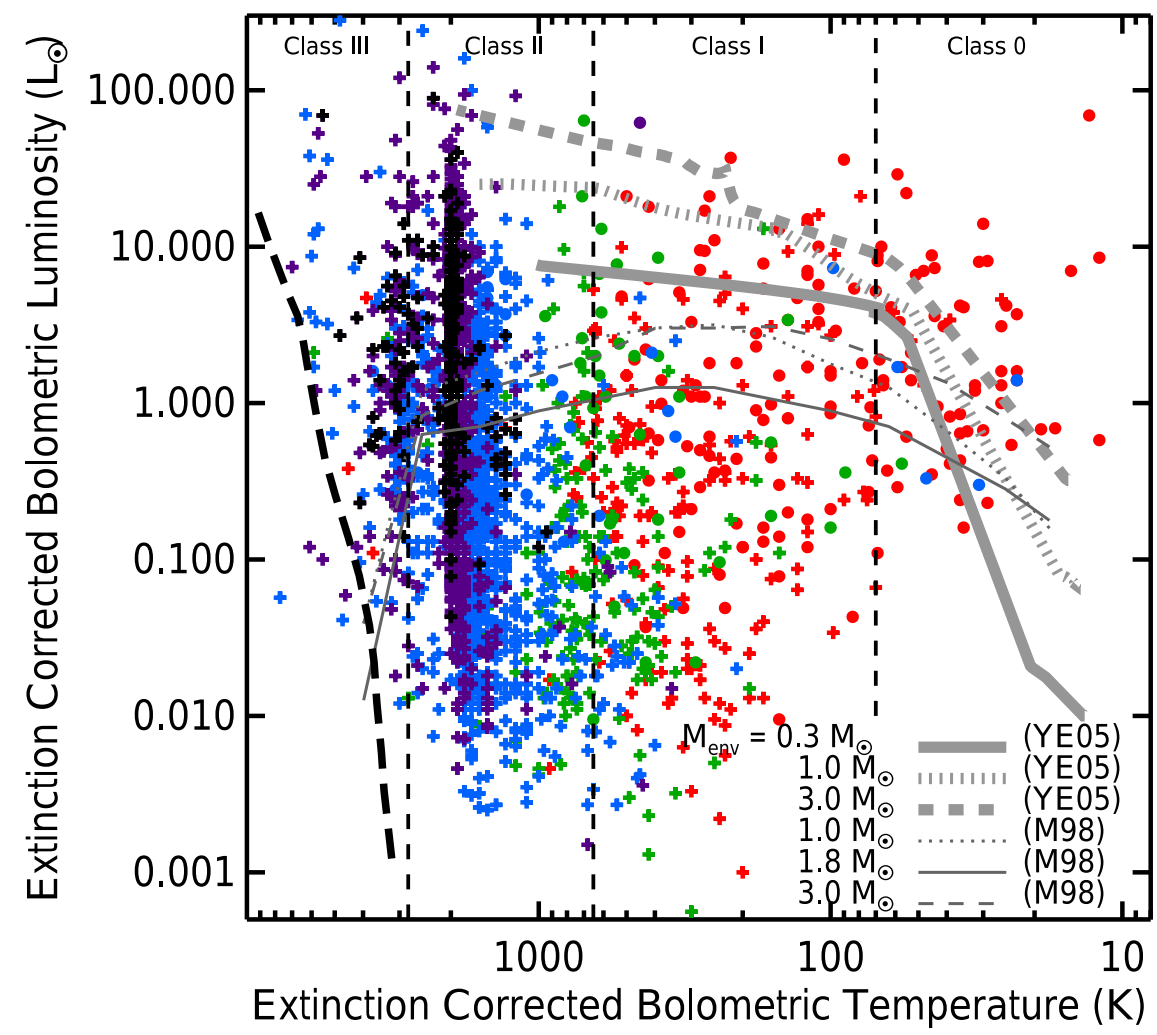

Figure 4. Extinction corrected bolometric luminosity $\left(L_{\mathrm{bol}}^{\prime}\right)$ plotted vs. the extinction corrected bolometric temperature $\left(T_{\mathrm{bol}}^{\prime}\right)$ for all $2966 \mathrm{YSOs}$. The color of each symbol shows its spectral class determined from $\alpha^{\prime}$ (the extinction corrected infrared spectral index), with red indicating Class $0+\mathrm{I}$, green indicating Flat-spectrum, blue indicating Class II, and purple indicating Class III. Black symbols indicate sources with [3.6]-[24] $\leqslant 1.5$ that are considered likely to be background AGB stars (see Section 2.3). Filled circles mark sources associated with submillimeter or millimeter continuum emission tracing dense cores (see text for details), while the plus signs mark sources with no such associations. The three thick lines plot model tracks from Young \& Evans (2005) for singular isothermal spheres of different initial masses undergoing inside-out collapse with $100 \%$ efficiency (all mass initially in the cores end up in the stars). These models end when infall stops and the cores have fully accreted onto the stars and are thus only showing evolution in the protostellar stage. The three thin lines plot model tracks from Myers et al. (1998) for cores of different initial masses collapsing with accretion rates that decrease exponentially with time and efficiencies less than one (i.e., only a fraction of the mass initially in the cores ends up in the stars, see Myers et al. 1998, for details). As above, these models are only relevant for the protostellar stage of evolution. The heavy dashed line on the left is the ZAMS from 0.1 to $2 M_{\odot}$ taken from D'Antona \& Mazzitelli (1994). The $T_{\text {bol }}$ class boundaries as defined by Chen et al. (1995) are plotted as vertical dashed lines.

with theoretical studies of the stages of the collapse of a dense, rotating core (Shu 1977; Terebey et al. 1984). Recent reviews on classification of young stars and the associations between observed SED classes and physical evolutionary stages are given by Evans et al. (2009) and Dunham et al. (2014).

Including the revisions by Greene et al. (1994), these empirical Classes are as follows:

1. Class $0+\mathrm{I}: \alpha^{\prime} \geqslant 0.3$

2. Flat-spectrum: $-0.3 \leqslant \alpha^{\prime}<0.3$;

3. Class II: $-1.6 \leqslant \alpha^{\prime}<-0.3$;

4. Class III: $\alpha^{\prime}<-1.6$.

We denote the infrared spectral index as $\alpha^{\prime}$ to emphasize that we only consider extinction corrected values in this study, and we refer to the first SED class as Class $0+\mathrm{I}$ instead of the more commonly used Class I to emphasize that Class 0 and I objects are both included in this definition (see below).

Table 5 lists, for each cloud, the numbers of YSOs in each Class using the extinction corrected infrared spectral indices, both with and without the likely background AGB stars removed. Summed over all clouds, out of the 2966 total YSOs, $326(11 \%)$ are classified as Class $0+\mathrm{I}, 210(7 \%)$ are classified as Flat-spectrum, $1248(42 \%)$ are classified as Class II, and $1182(40 \%)$ are classified as Class III. If we first remove the
298 sources identified as likely background AGB stars, out of the 2668 remaining YSOs, $326(12 \%)$ are classified as Class $0+$ I, $210(8 \%)$ are classified as Flat-spectrum, $1245(47 \%)$ are classified as Class II, and $887(33 \%)$ are classified as Class III. We emphasize that there are large variations between clouds in the relative numbers in each Class. To reinforce this point visually, Figures 5 and 6 plot, for each cloud, four-color bars where each color corresponds to a Class and the fractional length of each color represents the fraction of YSOs with the corresponding Class. Figure 5 displays the results including all 2966 YSOs whereas Figure 6 displays the results after removing the 298 likely background AGB stars. Finally, we also emphasize that our method of YSO identification is only capable of identifying Class III sources with detectable infrared excesses and is thus incomplete to the full populations of premain sequence stars in these clouds.

As noted above, we refer to the first Class as Class $0+\mathrm{I}$, but the original definition of this Class was simply Class I (Lada \& Wilking 1984; Greene et al. 1994). Class 0 objects were later added as an earlier Class for protostars too deeply embedded to detect in the infrared but inferred through other means (i.e., outflow presence; Andre et al. 1993). They are defined as objects with a ratio of submillimeter to bolometric luminosity $\left(L_{\mathrm{smm}} / L_{\mathrm{bol}}\right)$ greater than $0.5 \%$ (Andre et al. 1993), where $L_{\mathrm{smm}}$ is calculated for $\lambda \geqslant 350 \mu \mathrm{m}$. While they are defined 
Table 3

Observed Spitzer and 2MASS Flux Densities, in mJy, of the c2d+GB YSOs

\begin{tabular}{|c|c|c|c|c|c|c|c|c|c|c|c|c|c|c|c|c|c|c|}
\hline Index & $\begin{array}{c}J \\
1.25 \mu \mathrm{m}\end{array}$ & $\begin{array}{c}\Delta J \\
1.25 \mu \mathrm{m}\end{array}$ & $\begin{array}{c}H \\
1.65 \mu \mathrm{m}\end{array}$ & $\begin{array}{c}\Delta H \\
1.65 \mu \mathrm{m}\end{array}$ & $\begin{array}{c}K_{s} \\
2.17 \mu \mathrm{m}\end{array}$ & $\begin{array}{c}\Delta K_{s} \\
2.17 \mu \mathrm{m}\end{array}$ & $\begin{array}{c}\text { IRAC } \\
3.6 \mu \mathrm{m}\end{array}$ & $\begin{array}{l}\Delta \mathrm{IRAC} \\
3.6 \mu \mathrm{m}\end{array}$ & $\begin{array}{c}\text { IRAC } \\
4.5 \mu \mathrm{m}\end{array}$ & $\begin{array}{l}\Delta \mathrm{IRAC} \\
4.5 \mu \mathrm{m}\end{array}$ & $\begin{array}{c}\text { IRAC } \\
5.8 \mu \mathrm{m}\end{array}$ & $\begin{array}{l}\Delta \mathrm{IRAC} \\
5.8 \mu \mathrm{m}\end{array}$ & $\begin{array}{c}\text { IRAC } \\
8.0 \mu \mathrm{m}\end{array}$ & $\begin{array}{l}\Delta \mathrm{IRAC} \\
8.0 \mu \mathrm{m}\end{array}$ & $\begin{array}{l}\text { MIPS } \\
24 \mu \mathrm{m}\end{array}$ & $\begin{array}{c}\Delta \mathrm{MIPS} \\
24 \mu \mathrm{m}\end{array}$ & $\begin{array}{l}\text { MIPS } \\
70 \mu \mathrm{m}\end{array}$ & $\begin{array}{c}\Delta \mathrm{MIPS} \\
70 \mu \mathrm{m}\end{array}$ \\
\hline 1 & 290 & 7.3 & 820 & 33 & 1100 & 21 & 620 & 36 & 420 & 24 & 350 & 17 & 220 & 11 & 67 & 6.2 & $\ldots$ & $\ldots$ \\
\hline 2 & 140 & 3.3 & 370 & 14 & 480 & 9.3 & 300 & 15 & 160 & 7.7 & 150 & 6.9 & 92 & 4.3 & 36 & 3.3 & $\ldots$ & $\ldots$ \\
\hline 3 & 220 & 5.2 & 180 & 3.4 & 120 & 2.3 & 53 & 2.8 & 34 & 1.7 & 23 & 1.1 & 13 & 0.65 & 3.2 & 0.38 & 36 & 9.0 \\
\hline 4 & 340 & 9.9 & 890 & 36 & 1200 & 30 & 800 & 41 & 530 & 26 & 490 & 24 & 330 & 16 & 100 & 9.6 & $\ldots$ & $\ldots$ \\
\hline 5 & 37 & 1.0 & 40 & 1.1 & 30 & 0.83 & 13 & 0.64 & 8.8 & 0.42 & 5.9 & 0.28 & 3.5 & 0.17 & 1.5 & 0.22 & $\ldots$ & $\ldots$ \\
\hline 6 & 23 & 0.62 & 67 & 1.6 & 81 & 1.7 & 49 & 2.5 & 33 & 1.6 & 26 & 1.3 & 19 & 0.88 & 4.7 & 0.48 & $\ldots$ & $\ldots$ \\
\hline 7 & 88 & 1.9 & 200 & 4.8 & 250 & 4.9 & 140 & 7.4 & 96 & 4.7 & 77 & 3.7 & 50 & 2.4 & 14 & 1.3 & $\ldots$ & $\ldots$ \\
\hline 8 & 180 & 4.3 & 540 & 18 & 750 & 18 & 510 & 25 & 340 & 17 & 310 & 15 & 200 & 9.5 & 48 & 4.5 & $\ldots$ & $\ldots$ \\
\hline 9 & 200 & 4.8 & 520 & 16 & 700 & 16 & 410 & 21 & 230 & 11 & 200 & 9.6 & 120 & 5.8 & 40 & 3.7 & $\ldots$ & $\ldots$ \\
\hline 10 & 0.62 & 0.050 & 2.2 & 0.090 & 3.6 & 0.14 & 4.7 & 0.23 & 6.9 & 0.33 & 12 & 0.57 & 27 & 1.3 & 67 & 6.2 & 110 & 16 \\
\hline
\end{tabular}

(This table is available in its entirety in machine-readable form.) 
Table 4

Extinction Corrected Spitzer and 2MASS Flux Densities, in mJy, of the c2d+GB YSOs

\begin{tabular}{|c|c|c|c|c|c|c|c|c|c|c|c|c|c|c|c|c|c|c|}
\hline Index & $\begin{array}{c}J \\
1.25 \mu \mathrm{m}\end{array}$ & $\begin{array}{c}\Delta J \\
1.25 \mu \mathrm{m}\end{array}$ & $\begin{array}{c}H \\
1.25 \mu \mathrm{m}\end{array}$ & $\begin{array}{c}\Delta H \\
1.65 \mu \mathrm{m}\end{array}$ & $\begin{array}{c}K_{s} \\
2.17 \mu \mathrm{m}\end{array}$ & $\begin{array}{c}\Delta K_{s} \\
2.17 \mu \mathrm{m}\end{array}$ & $\begin{array}{c}\text { IRAC } \\
3.6 \mu \mathrm{m}\end{array}$ & $\begin{array}{l}\Delta \mathrm{IRAC} \\
3.6 \mu \mathrm{m}\end{array}$ & $\begin{array}{l}\text { IRAC } \\
4.5 \mu \mathrm{m}\end{array}$ & $\begin{array}{l}\Delta \mathrm{IRAC} \\
4.5 \mu \mathrm{m}\end{array}$ & $\begin{array}{c}\text { IRAC } \\
5.8 \mu \mathrm{m}\end{array}$ & $\begin{array}{l}\Delta \mathrm{IRAC} \\
5.8 \mu \mathrm{m}\end{array}$ & $\begin{array}{l}\text { IRAC } \\
8.0 \mu \mathrm{m}\end{array}$ & $\begin{array}{l}\Delta \mathrm{IRAC} \\
8.0 \mu \mathrm{m}\end{array}$ & $\begin{array}{l}\text { MIPS } \\
24 \mu \mathrm{m}\end{array}$ & $\begin{array}{l}\Delta \mathrm{MIPS} \\
24 \mu \mathrm{m}\end{array}$ & $\begin{array}{l}\text { MIPS } \\
70 \mu \mathrm{m}\end{array}$ & $\begin{array}{l}\Delta \mathrm{MIPS} \\
70 \mu \mathrm{m}\end{array}$ \\
\hline 1 & 2200 & 55 & 2800 & 120 & 2400 & 49 & 1000 & 59 & 630 & 35 & 490 & 23 & 320 & 16 & 82 & 7.6 & $\ldots$ & $\ldots$ \\
\hline 2 & 1000 & 24 & 1300 & 46 & 1100 & 21 & 490 & 25 & 240 & 12 & 200 & 9.6 & 130 & 6.3 & 43 & 4.0 & $\ldots$ & $\ldots$ \\
\hline 3 & 4700 & 110 & 1200 & 22 & 430 & 8.3 & 110 & 5.9 & 63 & 3.1 & 39 & 1.8 & 24 & 1.2 & 4.4 & 0.51 & 38 & 9.4 \\
\hline 4 & 2600 & 78 & 3200 & 130 & 2900 & 70 & 1300 & 68 & 800 & 40 & 680 & 34 & 490 & 23 & 130 & 12 & $\ldots$ & $\ldots$ \\
\hline 5 & 810 & 22 & 260 & 7.2 & 110 & 3.0 & 28 & 1.4 & 16 & 0.78 & 9.7 & 0.46 & 6.3 & 0.30 & 2.0 & 0.30 & $\ldots$ & $\ldots$ \\
\hline 6 & 170 & 4.5 & 220 & 5.4 & 190 & 3.9 & 79 & 4.0 & 49 & 2.3 & 37 & 1.7 & 27 & 1.3 & 5.7 & 0.58 & $\ldots$ & $\ldots$ \\
\hline 7 & 480 & 11 & 570 & 14 & 510 & 9.9 & 220 & 11 & 140 & 6.6 & 100 & 4.9 & 69 & 3.3 & 17 & 1.6 & $\ldots$ & $\ldots$ \\
\hline 8 & 1700 & 40 & 2100 & 69 & 1900 & 45 & 880 & 44 & 530 & 27 & 450 & 21 & 300 & 14 & 60 & 5.6 & $\ldots$ & $\ldots$ \\
\hline 9 & 1500 & 35 & 1800 & 53 & 1600 & 36 & 680 & 34 & 340 & 17 & 270 & 13 & 170 & 8.4 & 48 & 4.5 & $\ldots$ & $\ldots$ \\
\hline 10 & 13 & 1.1 & 14 & 0.59 & 13 & 0.50 & 10 & 0.49 & 13 & 0.61 & 20 & 0.95 & 48 & 2.2 & 90 & 8.4 & 110 & 17 \\
\hline
\end{tabular}

(This table is available in its entirety in machine-readable form.) 
Table 5

Number of YSOs by Cloud and Class

\begin{tabular}{|c|c|c|c|c|c|c|c|c|c|c|}
\hline \multirow[b]{2}{*}{ Cloud } & \multicolumn{5}{|c|}{ Extinction Corrected, All Sources } & \multicolumn{5}{|c|}{ Extinction Corrected, Excluding Likely AGB Stars } \\
\hline & $0+\mathrm{I}$ & Flat & II & III & Total & $0+\mathrm{I}$ & Flat & II & III & Total \\
\hline Aquila & 83 & 65 & 330 & 841 & 1319 & 83 & 65 & 327 & 634 & 1109 \\
\hline Auriga/CMC & 35 & 8 & 73 & 17 & 133 & 35 & 8 & 73 & 15 & 131 \\
\hline Cepheus & 18 & 11 & 61 & 13 & 103 & 18 & 11 & 61 & 12 & 102 \\
\hline Chamaeleon I & 4 & 5 & 62 & 15 & 86 & 4 & 5 & 62 & 14 & 85 \\
\hline Chamaeleon II & 2 & 0 & 19 & 5 & 26 & 2 & 0 & 19 & 4 & 25 \\
\hline Chamaeleon III & 1 & 0 & 0 & 3 & 4 & 1 & 0 & 0 & 0 & 1 \\
\hline Corona Australis & 9 & 6 & 22 & 17 & 54 & 9 & 6 & 22 & 16 & 53 \\
\hline IC5146 & 28 & 10 & 79 & 15 & 132 & 28 & 10 & 79 & 14 & 131 \\
\hline Lupus I & 1 & 2 & 8 & 2 & 13 & 1 & 2 & 8 & 0 & 11 \\
\hline Lupus III & 2 & 5 & 38 & 24 & 69 & 2 & 5 & 38 & 14 & 59 \\
\hline Lupus IV & 1 & 1 & 3 & 7 & 12 & 1 & 1 & 3 & 5 & 10 \\
\hline Lupus V & 0 & 0 & 7 & 36 & 43 & 0 & 0 & 7 & 25 & 32 \\
\hline Lupus VI & 1 & 0 & 2 & 42 & 45 & 1 & 0 & 2 & 25 & 28 \\
\hline Musca & 1 & 0 & 1 & 11 & 13 & 1 & 0 & 1 & 4 & 6 \\
\hline Ophiuchus & 28 & 43 & 174 & 47 & 292 & 28 & 43 & 174 & 32 & 277 \\
\hline Ophiuchus North & 2 & 1 & 3 & 4 & 10 & 2 & 1 & 3 & 3 & 9 \\
\hline Perseus & 76 & 35 & 235 & 39 & 385 & 76 & 35 & 235 & 31 & 377 \\
\hline Serpens & 34 & 18 & 131 & 44 & 227 & 34 & 18 & 131 & 39 & 222 \\
\hline Total & 326 & 210 & 1248 & 1182 & 2966 & 326 & 210 & 1245 & 887 & 2668 \\
\hline
\end{tabular}

observationally, the intended corresponding physical definition of Class 0 objects are protostars with at least $50 \%$ of their total mass still residing in the surrounding, infalling core (Andre et al. 1993). An alternative observational definition is given in terms of the bolometric temperature as objects with $T_{\text {bol }}<70 \mathrm{~K}$ (Myers \& Ladd 1993; Chen et al. 1995). While $L_{\mathrm{smm}} / L_{\mathrm{bol}}$ is generally a better discriminator between Class 0 and Class I objects than $T_{\text {bol }}$, and is less sensitive to source geometry (e.g., Young \& Evans 2005; Dunham et al. 2010, 2014), it cannot be calculated without $350 \mu \mathrm{m}$ photometry. As we do not have $350 \mu \mathrm{m}$ photometry available for many of our YSOs, we only consider $T_{\text {bol }}$ in this study.

With the sensitivity of Spitzer, Class 0 sources are easily detected in the infrared (e.g., Noriega-Crespo et al. 2004; Young et al. 2004; Dunham et al. 2006, 2008; Jørgensen et al. 2006; Tobin et al. 2010). Figure 7 plots $\alpha^{\prime}$ versus $T_{\text {bol }}^{\prime}$ for all 2966 YSOs. In general, the two quantities are anti-correlated, although there are some notable exceptions. There are a small number of SED Class $0+$ I sources (plotted in red in Figure 7) with sufficiently high $T_{\text {bol }}^{\prime}$ values to be classified as Class II or III by $T_{\text {bol }}^{\prime}$. None of these objects are associated with dense cores and are likely simply more evolved YSOs viewed through large extinctions that are not fully extinction-corrected (recall that, for SED Class $0+$ I sources, only the average cloud extinction is accounted for). There are also a few SED Class II YSOs (plotted in blue in Figure 7) with sufficiently low $T_{\text {bol }}^{\prime}$ values to be classified as Class 0 by $T_{\text {bol }}^{\prime}$. These are likely true protostars viewed at nearly pole-on inclinations through outflow cavities (e.g., Whitney et al. 2003; Robitaille et al. 2006; Crapsi et al. 2008), although detailed follow-up study is required to rule out chance alignments between more evolved YSOs and dense cores.

While there is a general anti-correlation between $\alpha^{\prime}$ and $T_{\mathrm{bol}}^{\prime}$, this trend weakens for objects classified as Class $0+$ I by $\alpha^{\prime}$ (objects with $\alpha^{\prime} \geqslant 0.3$ ). With substantial overlap in $\alpha^{\prime}$ for objects classified as Class 0 or Class I by $T_{\text {bol }}^{\prime}$, it is clear that these two Classes overlap in their infrared spectral indices. These results confirm those presented previously by Enoch et al. (2009) and Evans et al. (2009) and indicate that midinfrared data alone are insufficient to separate Class 0 and Class I objects; instead, full SEDs are required.

Using our compiled SEDs, and restricting ourselves only to those YSOs associated with a dense core (both to restrict ourselves to confirmed protostars and to ensure available submillimeter or millimeter detections for reliable measurements of $T_{\text {bol }}^{\prime}$ ), our $T_{\text {bol }}^{\prime}$ measurements result in 65 Class 0 protostars and 129 Class I protostars. Thus, we find that approximately one-third $(65 /[65+129]=0.335)$ of protostars are classified as Class 0 , in agreement with previous results based on the smaller c2d-only sample (Enoch et al. 2009; Evans et al. 2009). While future work must revisit this ratio once complete, sensitive submillimeter coverage is available for all clouds through the Herschel and JCMT GB surveys, preliminary Herschel results suggest only modest increases to the number of Class 0 objects (generally 15\%-30\%, Harvey et al. 2013; Stutz et al. 2013; Sadavoy et al. 2014), leading to only small upward revisions in the ratio of Class 0 to Class I protostars.

\subsection{Infrared Color-Color Diagrams}

Figure 8 presents an extinction corrected infrared color-color diagram of all YSOs identified in this work constructed from the four IRAC wavelengths: [3!6] - [4!5] plotted versus [5!8] - [8!.0]. The different Classes of YSOs (classified via $\alpha^{\prime}$ ) are generally well-separated in this diagram, as first noted by Allen et al. (2004). In cases where photometry is only available at $3.6-8.0 \mu \mathrm{m}$ and thus the infrared spectral index cannot be reliably calculated, this diagram can be used to approximately classify YSOs. Indeed, Allen et al. (2004) used the following two conditions to define a box where Class II sources are located:

$$
\text { 1. } 0.4 \leqslant[5.8]-[8.0] \leqslant 1.1 \text {, and }
$$$$
\text { 2. } 0.0 \leqslant[3.6]-[4.5] \leqslant 0.8 \text {. }
$$

The original definition of this "Class II box" by Allen et al. (2004) used observed photometry. Inspection of Figure 8 


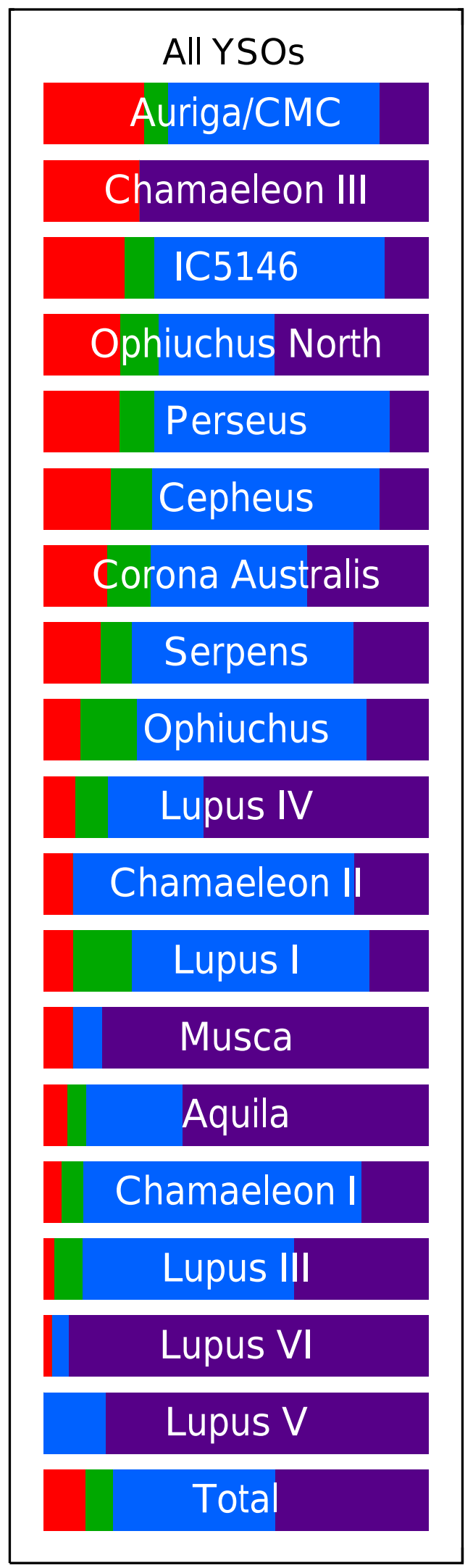

Figure 5. Four-color bar is plotted for each cloud, where each color corresponds to an evolutionary Class (similar to Figure 4, red corresponds to Class $0+\mathrm{I}$, green corresponds to Flat-spectrum, blue corresponds to Class II, and purple corresponds to Class III). The fractional length of each color represents the fraction of YSOs in each cloud with the corresponding Class. The clouds are sorted by Class $0+\mathrm{I}$ fraction, with the largest fractions at the top and the smallest fractions at the bottom, and all 2966 YSOs are included.

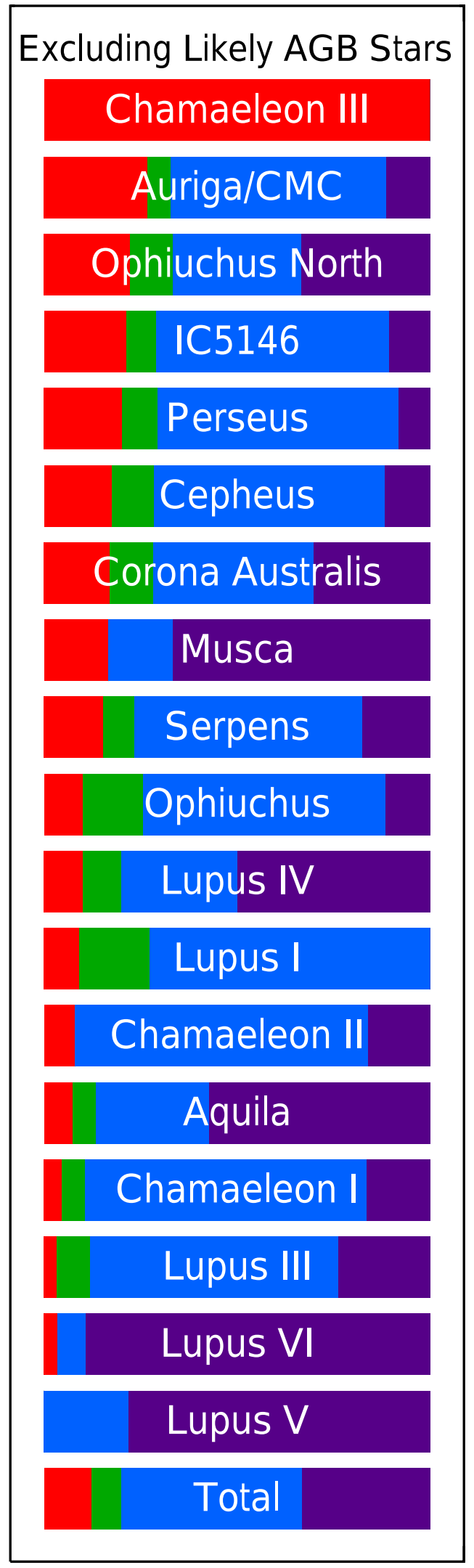

Figure 6. Same as Figure 5, except after removing the 298 objects identified as likely background AGB stars (see Section 2.3).

reveals that minor revisions are required when using extinction corrected photometry. While we acknowledge that polygons with greater than four sides could possibly give better results, 


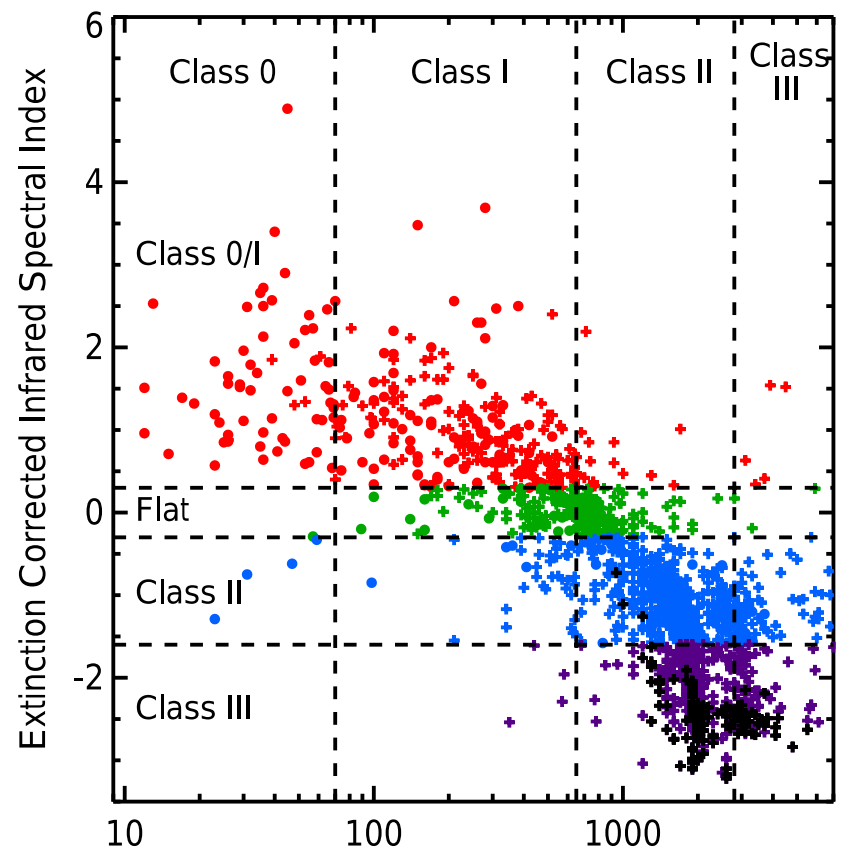

Extinction Corrected Bolometric Temperature (K)

Figure 7. Extinction corrected infrared spectral index $\left(\alpha^{\prime}\right)$ plotted vs. extinction corrected bolometric temperature $\left(T_{\text {bol }}^{\prime}\right)$ for all 2966 YSOs, with the colors and symbols the same as in Figure 4. The horizontal lines show the class boundaries in $\alpha^{\prime}$ as revised by Greene et al. (1994) and adopted in this work, and the vertical dashed lines show the $T_{\text {bol }}$ class boundaries as defined by Chen et al. (1995).

for simplicity we only consider rectangular regions. To define a new Class II box, we allow all four boundaries to vary and select those which simultaneously maximize the probability that a Class II YSO is located within the box (defined as the number of Class II YSOs located within the box divided by the total number of Class II YSOs) and the probability that a YSO located within the box is classified as Class II (defined as the number of Class II YSOs located within the box divided by the total number of YSOs located within the box). In practice, we implement these definitions by selecting the boundaries which maximize the sum of these two probabilities. Our proposed new Class II box, plotted in panel (b) of Figure 8, has the following boundaries:

1. $0.45 \leqslant[5 ! 8]-[8 ! 0] \leqslant 1.55$, and

2. $0.00 \leqslant[3 ! 6]-[4 ! 5] \leqslant 0.70$.

We note that the shape of this revised box is qualitatively similar to that proposed by Gutermuth (2005), who followed a similar procedure. With these revised boundaries, the fraction of Class II YSOs located within the box increases from 84.5\% to $89.9 \%$, and the fraction of YSOs in the box that are Class II increases from $78.8 \%$ to $82.4 \%$.

Figure 9 presents a second extinction corrected infrared color-color diagram of all YSOs which uses both the IRAC and MIPS observations by plotting [3!6] - [5!8] versus [8!0]$[24]^{\prime}$. Again the different Classes of YSOs (classified via $\alpha^{\prime}$ ) are generally well-separated, as first noted by Muzerolle et al. (2004). Indeed, Muzerolle et al. defined three regions in this color-color diagram to separate Class III/stellar, Class II, and Class $0+$ I YSOs; these regions are marked in panel (a) of Figure 9 and are defined in Table 6. As above, Muzerolle et al. used observed photometry, and we find that minor revisions are required when using extinction corrected photometry. Furthermore, the original boundaries defined by Muzerolle et al. did not include a region for Flat-spectrum objects. Thus, we follow the same procedure as above, again only considering rectangular regions, and maximize the sum of the eight probabilities (two for each Class), subject to the following constraints: (1) the minimum $x$ and $y$ Class III boundaries be held fixed at the Muzerolle et al. values, (2) there are no maximum $x$ and $y$ Class $0+\mathrm{I}$ boundaries, and (3) the regions can not overlap. Our revised boundaries are plotted in panel (b) of Figure 9 and listed in Table 6. Table 6 also lists each of the two probabilities considered for both the original and revised boundaries.

Ultimately, no region in either color-color diagram will classify YSOs with a perfect one-to-one correspondence to the infrared spectral index, but in cases where insufficient photometry at 2 and/or $24 \mu \mathrm{m}$ is available, approximate YSO classification is possible via infrared color-color diagrams.

\section{TIMESCALES FOR YSOS}

\subsection{Class $0+I$ and Flat-spectrum YSOs}

The standard method for determining the time spent in each YSO Class is to calculate the ratio of the number of sources in that Class to the number in a reference Class, and then multiply by the duration of the reference Class (e.g., Wilking et al. 1989; Evans et al. 2009; Dunham et al. 2014). Implicit in this method are the assumptions that star formation is continuous over at least the duration of the reference class and that time is the only variable. Regarding the first assumption, we strive to average out cloud-to-cloud variations in the recent star formation history by averaging over many clouds. Regarding the second, our results are best thought of as an average over all other relevant variables (e.g., environment, stellar mass; see Evans et al. 2009). In this section, we investigate the effects of different assumptions for the reference class and its duration on the timescales of Class $0+$ I and Flat-spectrum YSOs.

We first follow the method of Evans et al. (2009) and Dunham et al. (2014), who adopt Class II as the reference Class and assume a Class II duration of 2 Myr. This duration is taken from analyses of the fraction of cluster members with disks versus cluster age for various young clusters, which generally show a disk disperal time of a few Myr (Wyatt 2008; Ribas et al. 2014, 2015). As discussed extensively by Evans et al. (2009), this time is best considered as a disk half-life rather than an absolute duration. The first row of Table 7 presents durations for the Class $0+\mathrm{I}$ and Flat-spectrum Classes calculated following this method, using the extinction corrected numbers: 0.52 and $0.34 \mathrm{Myr}$ for Class $0+\mathrm{I}$ and Flat-spectrum objects, respectively.

Although the ages of young stars are highly uncertain, recent studies suggest a revised half-life for disk survival of 2.5-4 Myr on both observational (e.g., Kraus et al. 2012; Bell et al. 2013; Soderblom et al. 2014) and theoretical (e.g., Alexander \& Armitage 2009) grounds. Very recent results by Ribas et al. (2014, 2015), using spectroscopically confirmed members of various young clusters, show that this duration depends on the wavelength where the infrared excess indicative of the presence of a disk first appears, ranging from $1.9 \mathrm{Myr}$ for $\lambda<5 \mu \mathrm{m}$ to $3.0 \mathrm{Myr}$ for $\lambda \leqslant 24 \mu \mathrm{m}$ (note that these times have been converted from the $1 / e$ times presented by Ribas et al. to half-life times). As our Spitzer data are 

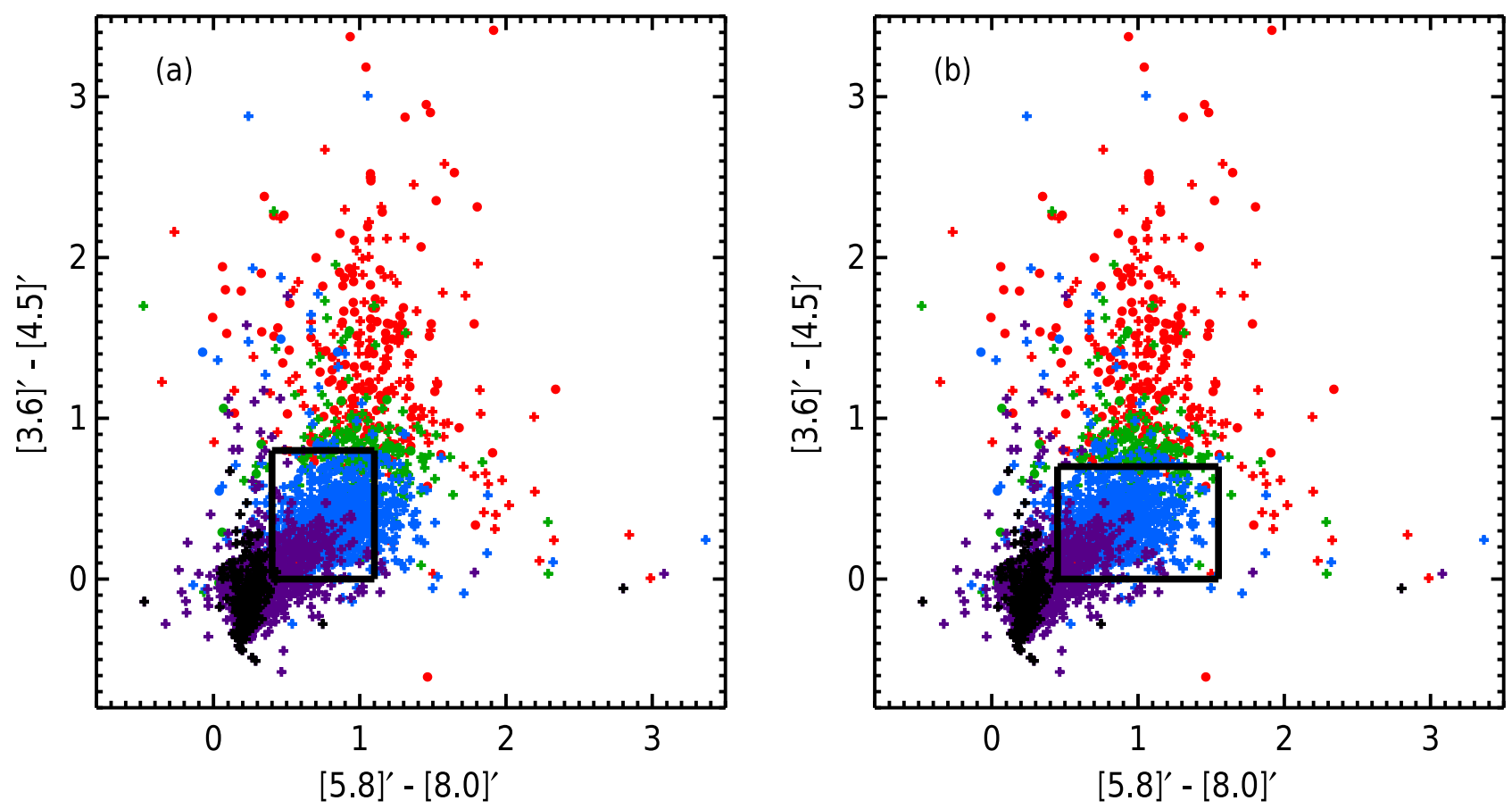

Figure 8. Extinction corrected color-color diagrams for the four IRAC bands, with the colors and symbols the same as in Figure 4. The box in (a) indicates the region identified with Class II sources by Allen et al. (2004), whereas in (b) it indicates our proposed modifications to this region (see text for details).

sensitive to infrared excesses out to $24 \mu \mathrm{m}$, and our Spitzer $\mathrm{c} 2 \mathrm{~d}+\mathrm{GB}$ data have comparable mid-infrared sensitivities to the Ribas et al. Spitzer and WISE data used to determine disk lifetimes, their derived lifetimes suggest that $3 \mathrm{Myr}$ is a more appropriate choice for the duration of the reference Class in this work. The second row of Table 7 lists the revised durations: 0.78 and $0.50 \mathrm{Myr}$ for Class $0+\mathrm{I}$ and Flat-spectrum objects, respectively.

While it has become somewhat standard to adopt Class II as the reference Class, this choice may result in a logical inconsistency. The duration of either 2 or $3 \mathrm{Myr}$ based on disk fraction versus cluster age is derived by determining the ratio of cluster members with infrared excesses to all cluster members (in other words, the ratio of disk sources to disk + no disk sources). By adopting Class II as the reference Class, we omit all of the Class III YSOs that still have disks, even though these objects are included when other studies (such as those by Ribas et al. ) derive disk lifetimes. Since infrared colors indicative of dust are the fundamental ingredient in our identifcation of YSOs (see Section 2.2), by definition the Class III YSOs identified in this work have disks (if they are all in fact YSOs; the issue of Class III contamination will be addressed in the following paragraph). Since the recent work by Ribas et al. $(2014,2015)$ is based on mid-infrared data from Spitzer and WISE with comparable sensitivity to midinfrared excesses as our c2d+GB data, and since we use disk lifetimes derived by Ribas et al. to set the duration of the reference Class, and further given that all Class II and Class III sources identified here have disks, we thus argue that it is more appropriate to adopt the sum of Class II and Class III as the reference Class than Class II alone. Thus, the third row of Table 7 lists the revised durations assuming a duration of $3 \mathrm{Myr}$ for the sum of Class II and III sources: 0.40 and $0.26 \mathrm{Myr}$ for Class $0+\mathrm{I}$ and Flat-spectrum objects, respectively.
The one major complication with the above analysis is the fact that our Class III sample suffers from $25 \%$ to $90 \%$ contamination, as discussed in Section 2.3. On the other hand, contamination is not a problem in the Ribas et al. (2014, 2015) samples since they only consider spectroscopically confirmed cluster members. As a result, the above calculations of Class durations likely overcount the number of objects in the reference Class and thus underestimate the Class $0+\mathrm{I}$ and Flat-spectrum durations. To account for this range of contamination rates, the last two columns of Table 7 list the calculated durations of the Class $0+\mathrm{I}$ and Flatspectrum Classes with the Class II + Class III reference Class corrected for contamination and a reference Class duration of $3 \mathrm{Myr}$. We consider these values to be our most accurate and logically consistent lifetimes: $0.46-0.72 \mathrm{Myr}$ for Class $0+$ I sources, and $0.30-0.46 \mathrm{Myr}$ for Flat-spectrum sources.

\subsection{Class 0 YSOs}

In Section 3.2, we reported that $33.5 \%$ of the objects associated with dense cores (and thus considered protostars according to the definition adopted in this paper) are classified as Class 0 by $T_{\text {bol }}^{\prime}$, with the remainder classified as Class I. Under the same assumptions as above (continuous star formation and time is the only variable) and our definition of a protostar as a YSO associated with a dense core, our results imply that $33.5 \%$ of the total protostellar duration is spent in the Class 0 phase, with the remaining $66.5 \%$ of the time spent in the Class I phase. If we assume that the lifetime of the SED Class $0+\mathrm{I}$ objects is a good proxy for the lifetime of the protostellar stage of evolution (see Section 4.3 below for justification of this assumption), the range of SED Class $0+$ I durations listed in Table 7 (0.40-0.78 Myr) imply resulting durations of $0.13-0.26 \mathrm{Myr}$ for Class 0 protostars and $0.27-0.52 \mathrm{Myr}$ for Class I protostars (see the last two columns 

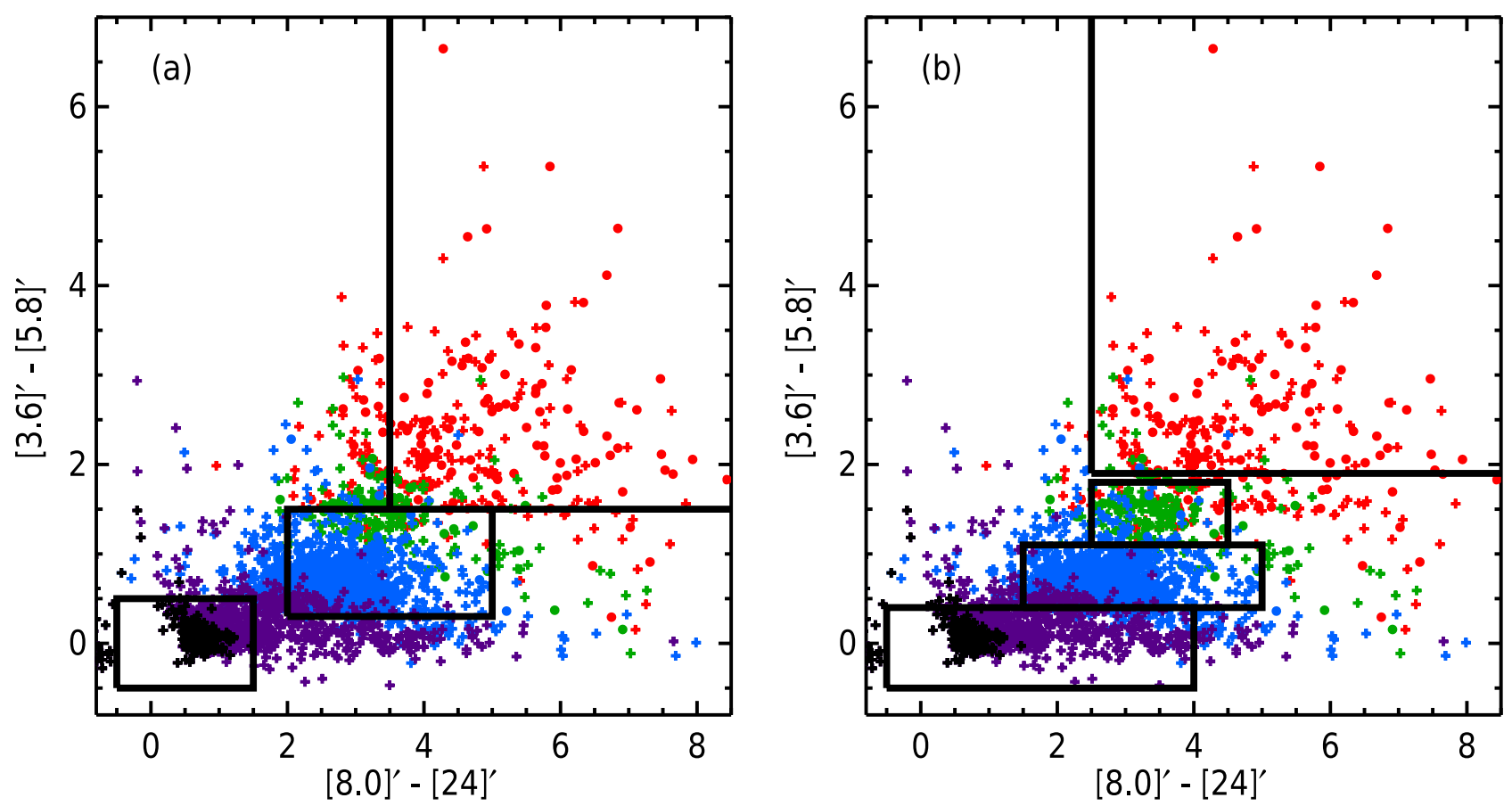

Figure 9. Extinction corrected color-color diagram using three of the four IRAC bands and the first MIPS band, with the colors and symbols the same as in Figure 4 . The boxes in (a) indicate the regions identified with Class III/stellar, Class II, and Class $0+$ I sources by Muzerolle et al. (2004), going from lower left to upper right. The boxes in (b) indicate our proposed modifications to these regions, now with four regions (Class III/stellar, Class II, Flat-spectrum, and Class $0+$ I, again from lower left to upper right).

Table 6

Classification Via [3!6] - [5! 8] vs. [8! 0$]-[24]^{\prime}$

\begin{tabular}{lcclc}
\hline \hline Quantity & Class III/stellar & Class II & Flat & Class 0 + I \\
\hline Original $\mathrm{x}_{\min }$ & -0.5 & 2.0 & $\ldots$ & 3.50 \\
Original $\mathrm{x}_{\max }$ & 1.5 & 5.0 & $\ldots$ & $\ldots$ \\
Original $\mathrm{y}_{\min }$ & -0.5 & 0.3 & $\ldots$ & 1.50 \\
Original $\mathrm{y}_{\max }$ & 0.5 & 1.5 & $\ldots$ & $\ldots$ \\
Original $\mathrm{P}_{1}^{\mathrm{a}}$ & $65.2 \%$ & $77.9 \%$ & $\ldots$ & $66.0 \%$ \\
Original $\mathrm{P}_{2}{ }^{\mathrm{b}}$ & $99.5 \%$ & $84.2 \%$ & $\ldots$ & $84.1 \%$ \\
Revised $\mathrm{x}_{\min }$ & -0.5 & 1.5 & 2.5 & 2.5 \\
Revised $\mathrm{x}_{\max }$ & 4.0 & 5.0 & 4.5 & $\ldots$ \\
Revised $\mathrm{y}_{\min }$ & -0.5 & 0.4 & 1.1 & 1.9 \\
Revised $\mathrm{y}_{\max }$ & 0.4 & 1.1 & 1.8 & $\ldots$ \\
Revised $\mathrm{P}_{1}{ }^{\mathrm{a}}$ & $83.2 \%$ & $79.2 \%$ & $63.2 \%$ & $65.6 \%$ \\
Revised $\mathrm{P}_{2}{ }^{\mathrm{b}}$ & $92.2 \%$ & $91.8 \%$ & $59.1 \%$ & $90.0 \%$ \\
\hline
\end{tabular}

Notes.

${ }^{a}$ The probability that a YSO of the desired Class is located within the region.

${ }^{\mathrm{b}}$ The probability that a YSO located within the region has the desired Class.

of Table 7). If we only consider the last two rows of Table 7 (including Class III YSOs in the reference Class, correcting for estimated contamination rates in the Class III sample, and adopting a reference Class duration of $3 \mathrm{Myr}$ ), the resulting durations are $0.15-0.24 \mathrm{Myr}$ for Class 0 and $0.31-0.48 \mathrm{Myr}$ for Class I.

As noted in Section 3.2, we generally find the same ratios of SED Class 0 to SED Class $0+$ I YSOs as in previous studies (Evans et al. 2009; Enoch et al. 2009; Dunham et al. 2014). While those studies derived a Class 0 duration of $0.15-0.17 \mathrm{Myr}$, our expanded range of possible durations is due to our expanded analysis of the contents and duration of the reference Class.
Table 7

Timescales for Young Stellar Objects

\begin{tabular}{lccccc}
\hline \hline Reference Class & $\begin{array}{c}\tau_{\text {ref }}{ }^{\mathrm{a}} \\
(\mathrm{Myr})\end{array}$ & $\begin{array}{c}\tau_{0+I} \mathrm{~b} \\
(\mathrm{Myr})\end{array}$ & $\begin{array}{c}\tau_{\mathrm{F}}{ }^{\mathrm{c}} \\
(\mathrm{Myr})\end{array}$ & $\begin{array}{c}\tau_{0}{ }^{\mathrm{d}} \\
(\mathrm{Myr})\end{array}$ & $\begin{array}{c}\tau_{\mathrm{I}}^{\mathrm{e}} \\
(\mathrm{Myr})\end{array}$ \\
\hline All Class II & 2.0 & 0.52 & 0.34 & 0.17 & 0.35 \\
All Class II & 3.0 & 0.78 & 0.50 & 0.26 & 0.52 \\
All Class II + All Class III & 3.0 & 0.40 & 0.26 & 0.13 & 0.27 \\
$\begin{array}{l}\text { All Class II + 75\% of } \\
\quad \text { Class III }\end{array}$ & 3.0 & 0.46 & 0.30 & 0.15 & 0.31 \\
$\begin{array}{l}\text { All Class II + 10\% of } \\
\quad \text { Class III }\end{array}$ & 3.0 & 0.72 & 0.46 & 0.24 & 0.48 \\
\hline
\end{tabular}

Notes.

${ }^{\text {a }}$ Assumed duration of the reference Class.

${ }^{\text {b }}$ Calculated duration of Class $0+$ I sources $\left(\tau_{\text {ref }} \frac{N_{0+1}}{N_{\text {ref }}}\right)$.

${ }^{\mathrm{c}}$ Calculated duration of Flat-spectrum sources $\left(\tau_{\text {ref }} \frac{N_{\mathrm{F}}}{N_{\text {ref }}}\right)$.

${ }^{\mathrm{d}}$ Calculated duration of Class 0 sources $\left(0.335 \times \tau_{0+\mathrm{I}}\right)$; see Section 4.2 for details.

${ }^{\mathrm{e}}$ Calculated duration of Class I sources $\left(0.665 \times \tau_{0+\mathrm{I}}\right)$; see Section 4.2 for details.

\subsection{Lifetimes of Protostars}

As noted in Section 3.2, the standard picture of low-mass star formation resulted from the merger of an empirical classification system based on the infrared spectral indices of YSOs (Lada \& Wilking 1984) with theoretical studies of the stages of the collapse of a dense, rotating core (Shu 1977; Terebey et al. 1984). In this picture, SED Class $0+$ I YSOs are protostars still embedded in and accreting from dense cores, SED Class II YSOs are T Tauri stars with optically thick, accreting disks, and SED Class III YSOs are pre-main sequence stars with little or no disks left (all Spitzer-identified Class III YSOs, by definition, still have infrared excesses, thus 
Table 8

Spitzer AORs for Unpublished Clouds

\begin{tabular}{|c|c|c|c|c|}
\hline Cloud & MIPS Target Name & MIPS AOR & IRAC Target Name & IRAC AOR \\
\hline \multirow[t]{49}{*}{ Aquila } & \multirow[t]{2}{*}{ SERAQU_E_1 } & 19968768 & SERAQU_E_1 & 20008448 \\
\hline & & 19968256 & $\ldots$ & 20008704 \\
\hline & \multirow[t]{2}{*}{ SERAQU_E_2 } & 20001536 & $\ldots$ & 20008960 \\
\hline & & 20001024 & $\ldots$ & 19970816 \\
\hline & \multirow[t]{2}{*}{ SERAQU_E_3 } & 19970560 & $\ldots$ & 19971072 \\
\hline & & 19970304 & $\ldots$ & 19971328 \\
\hline & SERAQU_E_4 & 20002048 & SERAQU_E_2 & 20005120 \\
\hline & \multirow{2}{*}{$\begin{array}{l}\ldots \\
\ldots\end{array}$} & 20001792 & $\ldots$ & 20005376 \\
\hline & & $\ldots$ & $\ldots$ & 20005632 \\
\hline & $\ldots$ & $\ldots$ & $\ldots$ & 19964672 \\
\hline & \multirow{2}{*}{$\ldots$} & $\ldots$ & $\ldots$ & 19965440 \\
\hline & & $\ldots$ & $\ldots$ & 19965952 \\
\hline & $\cdots$ & $\ldots$ & SERAQU_E_3 & 20002304 \\
\hline & $\ldots$ & $\ldots$ & $\ldots$ & 20002560 \\
\hline & $\cdots$ & $\ldots$ & $\ldots$ & 20003072 \\
\hline & $\ldots$ & $\cdots$ & $\cdots$ & 19957248 \\
\hline & $\begin{array}{l}\cdots \\
\cdots\end{array}$ & $\ldots$ & $\cdots$ & 19958016 \\
\hline & $\ldots$ & $\ldots$ & $\ldots$ & 19958272 \\
\hline & $\ldots$ & $\ldots$ & SERAQU_E_4 & 19997952 \\
\hline & $\ldots$ & $\ldots$ & $\ldots$ & 19998464 \\
\hline & $\ldots$ & $\ldots$ & $\ldots$ & 19998720 \\
\hline & $\ldots$ & $\ldots$ & $\ldots$ & 19999488 \\
\hline & $\ldots$ & $\ldots$ & $\ldots$ & 20018176 \\
\hline & $\ldots$ & $\ldots$ & $\ldots$ & 20018688 \\
\hline & $\ldots$ & $\ldots$ & $\ldots$ & 20018944 \\
\hline & $\ldots$ & $\ldots$ & $\ldots$ & 20019200 \\
\hline & $\ldots$ & $\ldots$ & SERAQU_E_5 & 14511104 \\
\hline & $\ldots$ & $\ldots$ & SERAQU_E_6 & 27042304 \\
\hline & $\ldots$ & $\ldots$ & $\ldots$ & 27042560 \\
\hline & SERAQU_NE & 14510336 & SERAQU_NE & 19991552 \\
\hline & $\ldots$ & 19966464 & $\ldots$ & 19988480 \\
\hline & $\ldots$ & 19966976 & $\ldots$ & 19991296 \\
\hline & $\ldots$ & $\ldots$ & $\ldots$ & 20009728 \\
\hline & $\ldots$ & $\ldots$ & $\ldots$ & 19987968 \\
\hline & & & $\ldots$ & 20009216 \\
\hline & SERAQU_SW_1 & 24195328 & SERAQU_SW & 19972352 \\
\hline & $\ldots$ & 24195584 & $\ldots$ & 19971840 \\
\hline & SERAQU_SW_2 & 24195840 & $\ldots$ & 19972608 \\
\hline & $\ldots$ & 24196096 & $\ldots$ & 19986944 \\
\hline & $\ldots$ & $\ldots$ & $\ldots$ & 19986688 \\
\hline & $\ldots$ & $\ldots$ & $\ldots$ & 19987200 \\
\hline & SERAQU_W_1 & 19974656 & SERAQU_W_1a & 19970048 \\
\hline & $\ldots$ & 19974400 & $\ldots$ & 19985664 \\
\hline & $\ldots$ & $\ldots$ & SERAQU_W_1b & 19962368 \\
\hline & $\ldots$ & $\ldots$ & & 19980800 \\
\hline & SERAQU_W_2 & 20006912 & SERAQU_W_2 & 19957760 \\
\hline & $\ldots$ & 20006656 & $\ldots$ & 19956992 \\
\hline & $\ldots$ & $\ldots$ & $\ldots$ & 19975424 \\
\hline & $\ldots$ & $\ldots$ & $\ldots$ & 19974912 \\
\hline Chamaeleon I & CHA_I & 03661312 & CHA_I_1 & 19986432 \\
\hline & $\ldots$ & 03962112 & $\ldots$ & 20006400 \\
\hline & $\ldots$ & 19979264 & CHA_I_2 & 03960320 \\
\hline & $\ldots$ & 20011008 & CHA_I_3 & 20015104 \\
\hline & $\ldots$ & 19978496 & $\ldots$ & 20014592 \\
\hline & $\ldots$ & 20010240 & CHA_I_4 & 03651328 \\
\hline & $\ldots$ & $\ldots$ & CHA_I_5 & 19992832 \\
\hline & $\ldots$ & $\ldots$ & $\ldots$ & 20012800 \\
\hline Chamaeleon III & CHA_III_1 & 19980032 & CHA_III_1 & 19995392 \\
\hline & $\ldots$ & 19979776 & $\ldots$ & 20015872 \\
\hline & CHA_III_2 & 20012544 & CHA_III_2a & 19989248 \\
\hline & $\ldots$ & 19977472 & $\ldots$ & 19989760 \\
\hline & $\ldots$ & 20012032 & $\ldots$ & 20007168 \\
\hline & $\ldots$ & 19976448 & $\ldots$ & 20007424 \\
\hline & $\cdots$ & $\ldots$ & CHA_III_2b & 19985152 \\
\hline
\end{tabular}


Table 8

(Continued)

\begin{tabular}{|c|c|c|c|c|}
\hline Cloud & MIPS Target Name & MIPS AOR & IRAC Target Name & IRAC AOR \\
\hline & $\ldots$ & $\ldots$ & $\ldots$ & 20003328 \\
\hline & $\ldots$ & $\ldots$ & CHA_III_2c & 19961344 \\
\hline & $\ldots$ & $\ldots$ & $\ldots$ & 19977216 \\
\hline & $\ldots$ & $\ldots$ & $\ldots$ & 19961600 \\
\hline & $\ldots$ & $\ldots$ & $\ldots$ & 19998208 \\
\hline & $\ldots$ & $\ldots$ & $\ldots$ & 20002816 \\
\hline & $\ldots$ & $\ldots$ & $\ldots$ & 27041536 \\
\hline & $\ldots$ & $\ldots$ & $\ldots$ & 27042048 \\
\hline \multirow[t]{6}{*}{ Musca } & MUSCA & 20004096 & MUSCA_1 & 27040768 \\
\hline & $\ldots$ & 19973120 & $\ldots$ & 27041024 \\
\hline & $\ldots$ & 20003840 & MUSCA_2 & 27043072 \\
\hline & $\ldots$ & 19972864 & $\ldots$ & 27043328 \\
\hline & $\ldots$ & $\ldots$ & $\ldots$ & 19975936 \\
\hline & $\ldots$ & $\ldots$ & $\ldots$ & 19977728 \\
\hline
\end{tabular}

the population of Class III YSOs without disks is not identified in the work presented here). Flat-spectrum YSOs have no clear link to a distinct physical stage, and in reality the correlation between observational SED Class and physical Stage is weakened by the effects of geometry, extinction, and accretion history (e.g., Whitney et al. 2003; Young \& Evans 2005; Robitaille et al. 2006; Crapsi et al. 2008; Dunham et al. 2010).

While our study provides the statistics necessary to measure durations of the observed SED Classes, it does not provide the necessary information to measure durations of physical Stages. In a separate, complementary study, Heiderman \& Evans (2015) surveyed nearly all of the SED Class $0+$ I and Flatspectrum YSOs in the c2d and GB surveys for emission from the dense gas tracer $\mathrm{HCO}^{+}(3-2)$. Using criteria based on the detection and strength of this line, they sorted objects into Stage $0+$ I (protostar) and Stage II (disk) sources. They found that the Flat-spectrum YSOs are approximately evenly divided between protostar and disk sources, with no evidence that they occupy a physically distinct evolutionary stage. They also found that the number of Flat-spectrum YSOs classified as protostars is balanced by the number of SED Class $0+$ I YSOs classified as disks, coincidentally resulting in a nearly identical number of protostars (defined in their work based on the presence of dense gas traced by $\mathrm{HCO}^{+}$line emission) and SED Class $0+$ I YSOs (326 Class $0+$ I YSOs identified here, 335 protostars identified by Heiderman \& Evans). Thus, based on their results, the range of possible protostellar stage durations is the same as the range of possible SED Class $0+$ I durations listed in Table 7.

\section{SUMMARY}

In this paper we have presented the full catalog of YSOs identified in the molecular clouds surveyed by the Spitzer Space Telescope "c2d" and "GB" Legacy surveys. In the case of the c2d clouds, our catalog updates a previous one published by Evans et al. (2009), and in the case of the GB clouds, our catalog represents the first catalog of Spitzer-identified YSOs in the full ensemble of these clouds. We summarize our main results as follows.

1. We have used standard techniques developed by the $\mathrm{c} 2 \mathrm{~d}$ project to identify 3239 candidate YSOs in the $18 \mathrm{c} 2 \mathrm{~d}$ and GB clouds, 2966 of which survive visual inspection and form our final catalog of YSOs in the GB.

2. We compile SEDs for all 2966 YSOs and correct these SEDs for foreground extinction.

3. We calculate and tabulate the infrared spectral index, bolometric luminosity, and bolometric temperature for each object, both with and without extinction corrections. We also tabulate information on whether or not each YSO is considered highly likely to be a background AGB star, and whether or not each YSO is associated with a dense core as traced by submillimeter or millimeter continuum emission.

4. After correcting for extinction, we find that 326 out of $2966(11 \%)$ are classified as Class 0 + I, 210 out of 2966 (7\%) are classified as Flat-spectrum, 1248 out of 2966 (42\%) are classified as Class II, and 1182 out of 2966 (40\%) are classified as Class III. The Class III sample suffers from an overall contamination rate by background AGB stars between $25 \%$ and $90 \%$.

5. Adopting standard assumptions, we derive durations of $0.40-0.78 \mathrm{Myr}$ for Class $0+$ I YSOs and $0.26-0.50 \mathrm{Myr}$ for Flat-spectrum YSOs, with most likely durations of 0.46-0.72 Myr (Class 0 + I) and 0.30-0.46 Myr (Flatspectrum). The ranges encompass uncertainties in the adopted assumptions.

6. Including information from submillimeter and millimeter wavelengths, one-third of the Class $0+\mathrm{I}$ sample is classified as Class 0. With this result, we calculate durations of $0.13-0.26 \mathrm{Myr}$ (Class 0) and $0.27-0.52 \mathrm{Myr}$ (Class I), with most likely durations of $0.15-0.24 \mathrm{Myr}$ (Class 0) and 0.31-0.48 Myr (Class I). As above, the ranges encompass uncertainties in the adopted assumptions. 
Table 9

Observed 0.36-0.96 $\mu$ m Flux Densities, in mJy, of the c2d+GB YSOs

\begin{tabular}{|c|c|c|c|c|c|c|c|c|c|c|c|c|c|c|c|c|}
\hline Index & $\begin{array}{c}U \\
0.36 \mu \mathrm{m}\end{array}$ & $\begin{array}{c}\Delta U \\
0.36 \mu \mathrm{m}\end{array}$ & $\begin{array}{c}B \\
0.44 \mu \mathrm{m}\end{array}$ & $\begin{array}{c}\Delta B \\
0.44 \mu \mathrm{m}\end{array}$ & $\begin{array}{c}V \\
0.55 \mu \mathrm{m}\end{array}$ & $\begin{array}{c}\Delta V \\
0.55 \mu \mathrm{m}\end{array}$ & $\begin{array}{c}R \\
0.64 \mu \mathrm{m}\end{array}$ & $\begin{array}{c}\Delta R \\
0.64 \mu \mathrm{m}\end{array}$ & $\begin{array}{c}H \alpha 12 \\
0.665 \mu \mathrm{m}\end{array}$ & $\begin{array}{c}\Delta H \alpha 12 \\
0.665 \mu \mathrm{m}\end{array}$ & $\begin{array}{c}I \\
0.79 \mu \mathrm{m}\end{array}$ & $\begin{array}{c}\Delta I \\
0.79 \mu \mathrm{m}\end{array}$ & $\begin{array}{c}m 914 \\
0.915 \mu \mathrm{m}\end{array}$ & $\begin{array}{c}\Delta m 914 \\
0.915 \mu \mathrm{m}\end{array}$ & $\begin{array}{c}z \\
0.96 \mu \mathrm{m}\end{array}$ & $\begin{array}{c}\Delta z \\
0.96 \mu \mathrm{m}\end{array}$ \\
\hline 1 & $\ldots$ & $\ldots$ & $\ldots$ & $\ldots$ & $\ldots$ & $\ldots$ & $\ldots$ & $\ldots$ & $\ldots$ & $\ldots$ & $\ldots$ & $\ldots$ & $\ldots$ & $\ldots$ & $\ldots$ & $\ldots$ \\
\hline 2 & $\ldots$ & $\ldots$ & $\ldots$ & $\ldots$ & $\ldots$ & $\ldots$ & $\ldots$ & $\ldots$ & $\ldots$ & $\ldots$ & $\ldots$ & $\ldots$ & $\ldots$ & $\ldots$ & $\ldots$ & $\ldots$ \\
\hline 3 & $\ldots$ & $\ldots$ & $\ldots$ & $\ldots$ & $\ldots$ & $\ldots$ & $\ldots$ & $\ldots$ & $\ldots$ & $\ldots$ & $\ldots$ & $\ldots$ & $\ldots$ & $\ldots$ & $\ldots$ & $\ldots$ \\
\hline 4 & $\ldots$ & $\ldots$ & $\ldots$ & $\ldots$ & $\ldots$ & $\ldots$ & $\ldots$ & $\ldots$ & $\ldots$ & $\ldots$ & $\ldots$ & $\ldots$ & $\ldots$ & $\ldots$ & $\ldots$ & $\ldots$ \\
\hline 5 & $\ldots$ & $\ldots$ & $\ldots$ & $\ldots$ & $\ldots$ & $\ldots$ & $\ldots$ & $\ldots$ & $\ldots$ & $\ldots$ & $\ldots$ & $\ldots$ & $\ldots$ & $\ldots$ & $\ldots$ & $\ldots$ \\
\hline 6 & $\ldots$ & $\ldots$ & $\ldots$ & $\ldots$ & $\ldots$ & $\ldots$ & $\ldots$ & $\ldots$ & $\ldots$ & $\ldots$ & $\ldots$ & $\ldots$ & $\ldots$ & $\ldots$ & $\ldots$ & $\ldots$ \\
\hline 7 & $\ldots$ & $\ldots$ & $\ldots$ & $\ldots$ & $\ldots$ & $\ldots$ & $\ldots$ & $\ldots$ & $\ldots$ & $\ldots$ & $\ldots$ & $\ldots$ & $\ldots$ & $\ldots$ & $\ldots$ & $\ldots$ \\
\hline 8 & $\ldots$ & $\ldots$ & $\ldots$ & $\ldots$ & $\ldots$ & $\ldots$ & $\ldots$ & $\ldots$ & $\ldots$ & $\ldots$ & $\ldots$ & $\ldots$ & $\ldots$ & $\ldots$ & $\ldots$ & $\ldots$ \\
\hline 9 & $\ldots$ & $\ldots$ & $\ldots$ & $\ldots$ & $\ldots$ & $\ldots$ & $\ldots$ & $\ldots$ & $\ldots$ & $\ldots$ & $\ldots$ & $\ldots$ & $\ldots$ & $\ldots$ & $\ldots$ & $\ldots$ \\
\hline 10 & $\ldots$ & $\ldots$ & $\ldots$ & $\ldots$ & $\ldots$ & $\ldots$ & $\ldots$ & $\ldots$ & $\ldots$ & $\ldots$ & $\ldots$ & $\ldots$ & $\ldots$ & $\ldots$ & $\ldots$ & $\ldots$ \\
\hline
\end{tabular}

(This table is available in its entirety in machine-readable form.) 
Table 10

Extinction Corrected 0.36-0.96 $\mu$ m Flux Densities, in mJy, of the c2d+GB YSOs

\begin{tabular}{|c|c|c|c|c|c|c|c|c|c|c|c|c|c|c|c|c|}
\hline Index & $\begin{array}{c}U \\
0.36 \mu \mathrm{m}\end{array}$ & $\begin{array}{c}\Delta U \\
0.36 \mu \mathrm{m}\end{array}$ & $\begin{array}{c}B \\
0.44 \mu \mathrm{m}\end{array}$ & $\begin{array}{c}\Delta B \\
0.44 \mu \mathrm{m}\end{array}$ & $\begin{array}{c}V \\
0.55 \mu \mathrm{m}\end{array}$ & $\begin{array}{c}\Delta V \\
0.55 \mu \mathrm{m}\end{array}$ & $\begin{array}{c}R \\
0.64 \mu \mathrm{m}\end{array}$ & $\begin{array}{c}\Delta R \\
0.64 \mu \mathrm{m}\end{array}$ & $\begin{array}{c}H \alpha 12 \\
0.665 \mu \mathrm{m}\end{array}$ & $\begin{array}{c}\Delta H \alpha 12 \\
0.665 \mu \mathrm{m}\end{array}$ & $\begin{array}{c}I \\
0.79 \mu \mathrm{m}\end{array}$ & $\begin{array}{c}\Delta I \\
0.79 \mu \mathrm{m}\end{array}$ & $\begin{array}{c}m 914 \\
0.915 \mu \mathrm{m}\end{array}$ & $\begin{array}{c}\Delta m 914 \\
0.915 \mu \mathrm{m}\end{array}$ & $\begin{array}{c}z \\
0.96 \mu \mathrm{m}\end{array}$ & $\begin{array}{c}\Delta z \\
0.96 \mu \mathrm{m}\end{array}$ \\
\hline 1 & $\ldots$ & $\ldots$ & $\ldots$ & $\ldots$ & $\ldots$ & $\ldots$ & $\ldots$ & $\ldots$ & $\ldots$ & $\ldots$ & $\ldots$ & $\ldots$ & $\ldots$ & $\ldots$ & $\ldots$ & $\ldots$ \\
\hline 2 & $\ldots$ & $\ldots$ & $\ldots$ & $\ldots$ & $\ldots$ & $\ldots$ & $\ldots$ & $\ldots$ & $\ldots$ & $\ldots$ & $\ldots$ & $\ldots$ & $\ldots$ & $\ldots$ & $\ldots$ & $\ldots$ \\
\hline 3 & $\ldots$ & $\ldots$ & $\ldots$ & $\ldots$ & $\ldots$ & $\ldots$ & $\ldots$ & $\ldots$ & $\ldots$ & $\ldots$ & $\ldots$ & $\ldots$ & $\ldots$ & $\ldots$ & $\ldots$ & $\ldots$ \\
\hline 4 & $\ldots$ & $\ldots$ & $\ldots$ & $\ldots$ & $\ldots$ & $\ldots$ & $\ldots$ & $\ldots$ & $\ldots$ & $\ldots$ & $\ldots$ & $\ldots$ & $\ldots$ & $\ldots$ & $\ldots$ & $\ldots$ \\
\hline 5 & $\ldots$ & $\ldots$ & $\ldots$ & $\ldots$ & $\ldots$ & $\ldots$ & $\ldots$ & $\ldots$ & $\ldots$ & $\ldots$ & $\ldots$ & $\ldots$ & $\ldots$ & $\ldots$ & $\ldots$ & $\ldots$ \\
\hline 6 & $\ldots$ & $\ldots$ & $\ldots$ & $\ldots$ & $\ldots$ & $\ldots$ & $\ldots$ & $\ldots$ & $\ldots$ & $\ldots$ & $\ldots$ & $\ldots$ & $\ldots$ & $\ldots$ & $\ldots$ & $\ldots$ \\
\hline 7 & $\ldots$ & $\ldots$ & $\ldots$ & $\ldots$ & $\ldots$ & $\ldots$ & $\ldots$ & $\ldots$ & $\ldots$ & $\ldots$ & $\ldots$ & $\ldots$ & $\ldots$ & $\ldots$ & $\ldots$ & $\ldots$ \\
\hline 8 & $\ldots$ & $\ldots$ & $\ldots$ & $\ldots$ & $\ldots$ & $\ldots$ & $\ldots$ & $\ldots$ & $\ldots$ & $\ldots$ & $\ldots$ & $\ldots$ & $\ldots$ & $\ldots$ & $\ldots$ & $\ldots$ \\
\hline 9 & $\ldots$ & $\ldots$ & $\ldots$ & $\ldots$ & $\ldots$ & $\ldots$ & $\ldots$ & $\ldots$ & $\ldots$ & $\ldots$ & $\ldots$ & $\ldots$ & $\ldots$ & $\ldots$ & $\ldots$ & $\ldots$ \\
\hline 10 & $\ldots$ & $\ldots$ & $\ldots$ & $\ldots$ & $\ldots$ & $\ldots$ & $\ldots$ & $\ldots$ & $\ldots$ & $\ldots$ & $\ldots$ & $\ldots$ & $\ldots$ & $\ldots$ & $\ldots$ & $\ldots$ \\
\hline
\end{tabular}

(This table is available in its entirety in machine-readable form.) 
Table 11

Observed 3.4-22 $\mu$ m Flux Densities, in mJy, of the c2d+GB YSOs

\begin{tabular}{|c|c|c|c|c|c|c|c|c|c|c|c|c|}
\hline Index & $\begin{array}{c}L \\
3.4 \mu \mathrm{m}\end{array}$ & $\begin{array}{c}\Delta L \\
3.4 \mu \mathrm{m}\end{array}$ & $\begin{array}{c}M \\
5.0 \mu \mathrm{m}\end{array}$ & $\begin{array}{c}\Delta M \\
5.0 \mu \mathrm{m}\end{array}$ & $\begin{array}{c}I S O \\
6.7 \mu \mathrm{m}\end{array}$ & $\begin{array}{c}\Delta I S O \\
6.7 \mu \mathrm{m}\end{array}$ & $\begin{array}{c}\text { WISE } \\
12 \mu \mathrm{m}\end{array}$ & $\begin{array}{c}\Delta W I S E \\
12 \mu \mathrm{m}\end{array}$ & $\begin{array}{c}I S O \\
14 \mu \mathrm{m}\end{array}$ & $\begin{array}{c}\Delta I S O \\
14 \mu \mathrm{m}\end{array}$ & $\begin{array}{c}\text { WISE } \\
22 \mu \mathrm{m}\end{array}$ & $\begin{array}{c}\Delta W I S E \\
22 \mu \mathrm{m}\end{array}$ \\
\hline 1 & $\ldots$ & $\ldots$ & $\ldots$ & $\ldots$ & $\ldots$ & $\ldots$ & 160 & 2.3 & $\ldots$ & $\ldots$ & 87 & 2.3 \\
\hline 2 & $\ldots$ & $\ldots$ & $\ldots$ & $\ldots$ & $\ldots$ & $\ldots$ & 70 & 1.0 & $\ldots$ & $\ldots$ & 47 & 1.6 \\
\hline 3 & $\ldots$ & $\ldots$ & $\ldots$ & $\ldots$ & $\ldots$ & $\ldots$ & 7.6 & 0.21 & $\ldots$ & $\ldots$ & 3.7 & 0.99 \\
\hline 4 & $\ldots$ & $\ldots$ & $\ldots$ & $\ldots$ & $\ldots$ & $\ldots$ & 240 & 3.3 & $\ldots$ & $\ldots$ & 120 & 2.9 \\
\hline 5 & $\ldots$ & $\ldots$ & $\ldots$ & $\ldots$ & $\ldots$ & $\ldots$ & 1.7 & 0.16 & $\ldots$ & $\ldots$ & $\ldots$ & $\ldots$ \\
\hline 6 & $\ldots$ & $\ldots$ & $\ldots$ & $\ldots$ & $\ldots$ & $\ldots$ & 14 & 0.28 & $\ldots$ & $\ldots$ & 8.2 & 0.85 \\
\hline 7 & $\ldots$ & $\ldots$ & $\ldots$ & $\ldots$ & $\ldots$ & $\ldots$ & 33 & 0.51 & $\ldots$ & $\ldots$ & 19 & 1.1 \\
\hline 8 & $\ldots$ & $\ldots$ & $\ldots$ & $\ldots$ & $\ldots$ & $\ldots$ & 120 & 1.5 & $\ldots$ & $\ldots$ & 63 & 2.1 \\
\hline 9 & $\ldots$ & $\ldots$ & $\ldots$ & $\ldots$ & $\ldots$ & $\ldots$ & 81 & 1.2 & $\ldots$ & $\ldots$ & 50 & 1.7 \\
\hline 10 & $\ldots$ & $\ldots$ & $\ldots$ & $\ldots$ & $\ldots$ & $\ldots$ & 27 & 0.47 & $\ldots$ & $\ldots$ & 65 & 2.2 \\
\hline
\end{tabular}

(This table is available in its entirety in machine-readable form.)

Table 12

Extinction Corrected 3.4-22 $\mu \mathrm{m}$ Flux Densities, in mJy, of the c2d+GB YSOs

\begin{tabular}{|c|c|c|c|c|c|c|c|c|c|c|c|c|}
\hline Index & $\begin{array}{c}L \\
3.4 \mu \mathrm{m}\end{array}$ & $\begin{array}{c}\Delta L \\
3.4 \mu \mathrm{m}\end{array}$ & $\begin{array}{c}M \\
5.0 \mu \mathrm{m}\end{array}$ & $\begin{array}{c}\Delta M \\
5.0 \mu \mathrm{m}\end{array}$ & $\begin{array}{c}I S O \\
6.7 \mu \mathrm{m}\end{array}$ & $\begin{array}{c}\Delta I S O \\
6.7 \mu \mathrm{m}\end{array}$ & $\begin{array}{c}\text { WISE } \\
12 \mu \mathrm{m}\end{array}$ & $\begin{array}{c}\Delta W I S E \\
12 \mu \mathrm{m}\end{array}$ & $\begin{array}{c}I S O \\
14 \mu \mathrm{m}\end{array}$ & $\begin{array}{c}\Delta I S O \\
14 \mu \mathrm{m}\end{array}$ & $\begin{array}{c}\text { WISE } \\
22 \mu \mathrm{m}\end{array}$ & $\begin{array}{c}\Delta W I S E \\
22 \mu \mathrm{m}\end{array}$ \\
\hline 1 & $\ldots$ & $\ldots$ & $\ldots$ & $\ldots$ & $\ldots$ & $\ldots$ & 230 & 3.4 & $\ldots$ & $\ldots$ & 110 & 2.9 \\
\hline 2 & $\ldots$ & $\ldots$ & $\ldots$ & $\ldots$ & $\ldots$ & $\ldots$ & 100 & 1.5 & $\ldots$ & $\ldots$ & 59 & 2.0 \\
\hline 3 & $\ldots$ & $\ldots$ & $\ldots$ & $\ldots$ & $\ldots$ & $\ldots$ & 14 & 0.39 & $\ldots$ & $\ldots$ & 5.2 & 1.4 \\
\hline 4 & $\ldots$ & $\ldots$ & $\ldots$ & $\ldots$ & $\ldots$ & $\ldots$ & 360 & 4.9 & $\ldots$ & $\ldots$ & 160 & 3.6 \\
\hline 5 & $\ldots$ & $\ldots$ & $\ldots$ & $\ldots$ & $\ldots$ & $\ldots$ & 3.1 & 0.29 & $\ldots$ & $\ldots$ & $\ldots$ & $\ldots$ \\
\hline 6 & $\ldots$ & $\ldots$ & $\ldots$ & $\ldots$ & $\ldots$ & $\ldots$ & 21 & 0.42 & $\ldots$ & $\ldots$ & 10 & 1.1 \\
\hline 7 & $\ldots$ & $\ldots$ & $\ldots$ & $\ldots$ & $\ldots$ & $\ldots$ & 46 & 0.72 & $\ldots$ & $\ldots$ & 23 & 1.3 \\
\hline 8 & $\ldots$ & $\ldots$ & $\ldots$ & $\ldots$ & $\ldots$ & $\ldots$ & 190 & 2.4 & $\ldots$ & $\ldots$ & 81 & 2.7 \\
\hline 9 & $\ldots$ & $\ldots$ & $\ldots$ & $\ldots$ & $\ldots$ & $\ldots$ & 120 & 1.8 & $\ldots$ & $\ldots$ & 62 & 2.1 \\
\hline 10 & $\ldots$ & $\ldots$ & $\ldots$ & $\ldots$ & $\ldots$ & $\ldots$ & 49 & 0.87 & $\ldots$ & $\ldots$ & 92 & 3.2 \\
\hline
\end{tabular}

(This table is available in its entirety in machine-readable form.)

7. We revisit infrared color-color diagrams used in the literature to classify YSOs and use our large, extinction corrected sample to propose revisions to classification boundaries in these diagrams.

8. The bolometric temperature of a YSO surrounded by a circumstellar disk but no longer embedded in a dense core is primarily determined by the spectral type of the YSO and the availability of optical photometry, and not by the properties of the circumstellar material. As such, the bolometric temperature should not be used to distinguish between Class II and Class III YSOs.

The YSO catalog presented here represents the most complete catalog of star formation within $500 \mathrm{pc}$ of the Sun yet assembled. It includes nearly every major site of star formation in the solar neighborhood except for the Taurus and Orion molecular clouds, each of which was the subject of its own, dedicated Spitzer Legacy survey. While this work represents a major step forward in assembling a complete and reliable catalog of YSOs in the GB, future work to further refine the YSO catalog presented here is needed on the following three fronts: (1) combining the results of the $\mathrm{c} 2 \mathrm{~d}+\mathrm{GB}$, Taurus, and Orion surveys using uniform data reduction and analysis techniques, (2) identifying and removing background AGB stars currently contaminating the sample, and (3) revisiting the associations between Spitzer sources and dense cores traced by submillimeter and millimeter detections once the full results from the Herschel and SCUBA-2 GB surveys are available.
We thank the referee for thoughtful comments that have improved the quality of this paper, and we acknowledge helpful discussions with H. Arce and L. Kristensen. This work is based primarily on observations obtained with the Spitzer Space Telescope, operated by the Jet Propulsion Laboratory, California Institute of Technology. It also uses data products from the 2MASS, which is a joint project of the University of Massachusetts and the Infrared Processing and Analysis Center/California Institute of Technology, funded by the National Aeronautics and Space Administration and the National Science Foundation. These data were provided by the NASA/IPAC Infrared Science Archive, which is operated by the Jet Propulsion Laboratory, California Institute of Technology, under contract with NASA. This research has made use of NASA's Astrophysics Data System (ADS) Abstract Service, the IDL Astronomy Library hosted by the NASA Goddard Space Flight Center, and the SIMBAD database operated at CDS, Strasbourg, France. M.M.D. acknowledges support from NASA ADAP grant NNX13AE54G and from the Submillimeter Array (SMA) through an SMA postdoctoral fellowship and from $\mathrm{H}$. Arce at Yale University, where a portion of this work was performed. N.J.E. is supported by NSF Grant AST-1109116 to the University of Texas at Austin. L.A.C. was supported by ALMA-CONICYT grant 31120009, CONICYT-FONDECYT grant 1140109, and the Millennium Science Initiative (Chilean Ministry of Economy) grant RC130007. A.L.H. is supported by an NSF AAPF under award AST-1302978. K.E.Y. acknowledges support from the 
Table 13

Observed $160-1300 \mu \mathrm{m}$ Flux Densities, in mJy, of the c2d+GB YSOs

\begin{tabular}{|c|c|c|c|c|c|c|c|c|c|c|c|c|c|c|}
\hline Index & $\begin{array}{c}M I P S \\
160 \mu \mathrm{m}\end{array}$ & $\begin{array}{l}\Delta M I P S \\
160 \mu \mathrm{m}\end{array}$ & $350 \mu \mathrm{m}$ & $\Delta 350 \mu \mathrm{m}$ & $450 \mu \mathrm{m}$ & $\Delta 450 \mu \mathrm{m}$ & $850 \mu \mathrm{m}$ & $\Delta 850 \mu \mathrm{m}$ & $1100 \mu \mathrm{m}$ & $\Delta 1100 \mu \mathrm{m}$ & $1200 \mu \mathrm{m}$ & $\Delta 1200 \mu \mathrm{m}$ & $1300 \mu \mathrm{m}$ & $\Delta 1300 \mu \mathrm{m}$ \\
\hline 1 & $\ldots$ & $\ldots$ & $\ldots$ & $\ldots$ & $\ldots$ & $\ldots$ & $\ldots$ & $\ldots$ & $\ldots$ & $\ldots$ & $\ldots$ & $\ldots$ & $\ldots$ & $\ldots$ \\
\hline 2 & $\ldots$ & $\ldots$ & $\ldots$ & $\ldots$ & $\ldots$ & $\ldots$ & $\ldots$ & $\ldots$ & $\ldots$ & $\ldots$ & $\ldots$ & $\ldots$ & $\ldots$ & $\ldots$ \\
\hline 3 & $\ldots$ & $\ldots$ & $\ldots$ & $\ldots$ & $\ldots$ & $\ldots$ & $\ldots$ & $\ldots$ & $\ldots$ & $\ldots$ & $\ldots$ & $\ldots$ & $\ldots$ & $\ldots$ \\
\hline 4 & $\ldots$ & $\ldots$ & $\ldots$ & $\ldots$ & $\ldots$ & $\ldots$ & $\ldots$ & $\ldots$ & $\ldots$ & $\ldots$ & $\ldots$ & $\ldots$ & $\ldots$ & $\ldots$ \\
\hline 5 & $\ldots$ & $\ldots$ & $\ldots$ & $\ldots$ & $\ldots$ & $\ldots$ & $\ldots$ & $\ldots$ & $\ldots$ & $\ldots$ & $\ldots$ & $\ldots$ & $\ldots$ & $\ldots$ \\
\hline 6 & $\ldots$ & $\ldots$ & $\ldots$ & $\ldots$ & $\ldots$ & $\ldots$ & $\ldots$ & $\ldots$ & $\ldots$ & $\ldots$ & $\ldots$ & $\ldots$ & $\ldots$ & $\ldots$ \\
\hline 7 & $\ldots$ & $\ldots$ & $\ldots$ & $\ldots$ & $\ldots$ & $\ldots$ & $\ldots$ & $\ldots$ & $\ldots$ & $\ldots$ & $\ldots$ & $\ldots$ & $\ldots$ & $\ldots$ \\
\hline 8 & $\ldots$ & $\ldots$ & $\ldots$ & $\ldots$ & $\ldots$ & $\ldots$ & $\ldots$ & $\ldots$ & $\ldots$ & $\ldots$ & $\ldots$ & $\ldots$ & $\ldots$ & $\ldots$ \\
\hline 9 & $\ldots$ & $\ldots$ & $\ldots$ & $\ldots$ & $\ldots$ & $\ldots$ & $\ldots$ & $\ldots$ & $\ldots$ & $\ldots$ & $\ldots$ & $\ldots$ & $\ldots$ & $\ldots$ \\
\hline 10 & $\ldots$ & $\ldots$ & $\ldots$ & $\ldots$ & $\ldots$ & $\ldots$ & $\ldots$ & $\ldots$ & $\ldots$ & $\ldots$ & $\ldots$ & $\ldots$ & $\ldots$ & $\ldots$ \\
\hline
\end{tabular}

(This table is available in its entirety in machine-readable form.) 
Table 14

Extinction Corrected 160-1300 $\mu$ m Flux Densities, in mJy, of the c2d+GB YSOs

\begin{tabular}{|c|c|c|c|c|c|c|c|c|c|c|c|c|c|c|}
\hline Index & $\begin{array}{c}M I P S \\
160 \mu \mathrm{m}\end{array}$ & $\begin{array}{l}\Delta M I P S \\
160 \mu \mathrm{m}\end{array}$ & $350 \mu \mathrm{m}$ & $\Delta 350 \mu \mathrm{m}$ & $450 \mu \mathrm{m}$ & $\Delta 450 \mu \mathrm{m}$ & $850 \mu \mathrm{m}$ & $\Delta 850 \mu \mathrm{m}$ & $1100 \mu \mathrm{m}$ & $\Delta 1100 \mu \mathrm{m}$ & $1200 \mu \mathrm{m}$ & $\Delta 1200 \mu \mathrm{m}$ & $1300 \mu \mathrm{m}$ & $\Delta 1300 \mu \mathrm{m}$ \\
\hline 1 & $\ldots$ & $\ldots$ & $\ldots$ & $\ldots$ & $\ldots$ & $\ldots$ & $\ldots$ & $\ldots$ & $\ldots$ & $\ldots$ & $\ldots$ & $\ldots$ & $\ldots$ & $\ldots$ \\
\hline 2 & $\ldots$ & $\ldots$ & $\ldots$ & $\ldots$ & $\ldots$ & $\ldots$ & $\ldots$ & $\ldots$ & $\ldots$ & $\ldots$ & $\ldots$ & $\ldots$ & $\ldots$ & $\ldots$ \\
\hline 3 & $\ldots$ & $\ldots$ & $\ldots$ & $\ldots$ & $\ldots$ & $\ldots$ & $\ldots$ & $\ldots$ & $\ldots$ & $\ldots$ & $\ldots$ & $\ldots$ & $\ldots$ & $\ldots$ \\
\hline 4 & $\ldots$ & $\ldots$ & $\ldots$ & $\ldots$ & $\ldots$ & $\ldots$ & $\ldots$ & $\ldots$ & $\ldots$ & $\ldots$ & $\ldots$ & $\ldots$ & $\ldots$ & $\ldots$ \\
\hline 5 & $\ldots$ & $\ldots$ & $\ldots$ & $\ldots$ & $\ldots$ & $\ldots$ & $\ldots$ & $\ldots$ & $\ldots$ & $\ldots$ & $\ldots$ & $\ldots$ & $\ldots$ & $\ldots$ \\
\hline 6 & $\ldots$ & $\ldots$ & $\ldots$ & $\ldots$ & $\ldots$ & $\ldots$ & $\ldots$ & $\ldots$ & $\ldots$ & $\ldots$ & $\ldots$ & $\ldots$ & $\ldots$ & $\ldots$ \\
\hline 7 & $\ldots$ & $\ldots$ & $\ldots$ & $\ldots$ & $\ldots$ & $\ldots$ & $\ldots$ & $\ldots$ & $\ldots$ & $\ldots$ & $\ldots$ & $\ldots$ & $\ldots$ & $\ldots$ \\
\hline 8 & $\ldots$ & $\ldots$ & $\ldots$ & $\ldots$ & $\ldots$ & $\ldots$ & $\ldots$ & $\ldots$ & $\ldots$ & $\ldots$ & $\ldots$ & $\ldots$ & $\cdots$ & $\ldots$ \\
\hline 9 & $\ldots$ & $\ldots$ & $\ldots$ & $\ldots$ & $\ldots$ & $\ldots$ & $\ldots$ & $\ldots$ & $\ldots$ & $\ldots$ & $\ldots$ & $\ldots$ & $\ldots$ & $\ldots$ \\
\hline 10 & $\ldots$ & $\ldots$ & $\ldots$ & $\ldots$ & $\ldots$ & $\ldots$ & $\ldots$ & $\ldots$ & $\ldots$ & $\ldots$ & $\ldots$ & $\ldots$ & $\ldots$ & $\ldots$ \\
\hline
\end{tabular}

(This table is available in its entirety in machine-readable form.) 


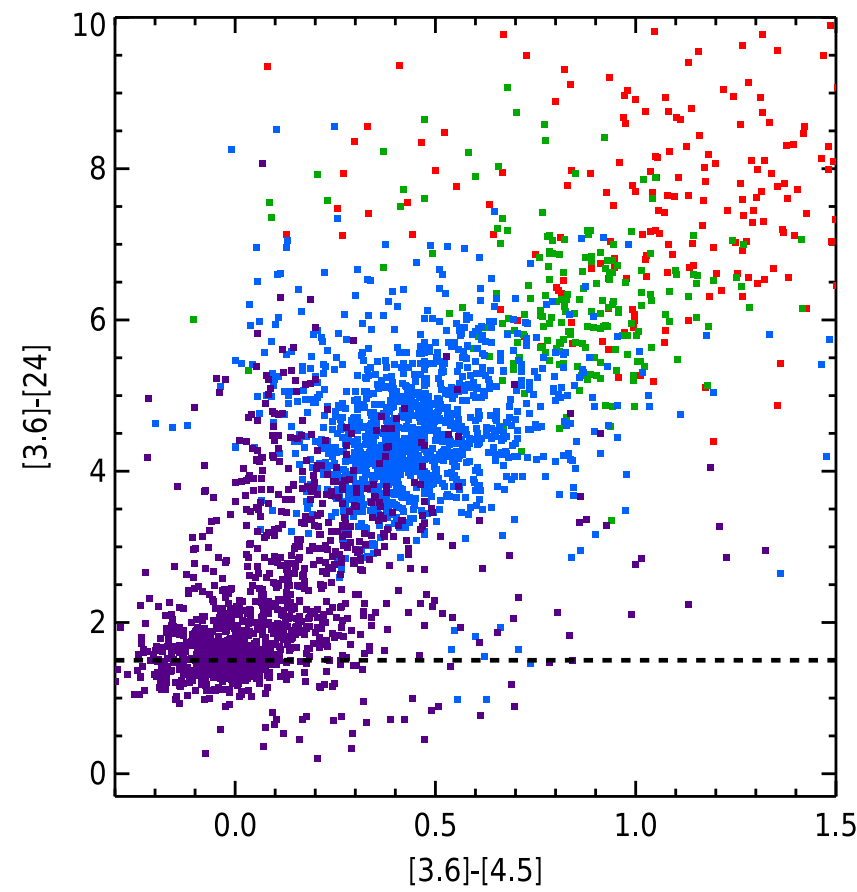

Figure 10. Same as Figure 2 ([3.6]-[24] vs. [3.6]-[4.5] for all 2966 objects in our final YSO sample, using the observed photometry), except plotted using the original data before rounding. The vertical line of sources at [3.6]-[4.5] 0.5 has now disappeared as YSOs with similar flux densities have not now all been rounded to identical flux densities.

Louisiana Space Consortium Research Enhancement Award through NASA EPSCoR grant No. NNX10AI40H. T.L.H. acknowledges support provided by NASA's Astrophysics Data Analysis Program, under grant No. NNX13AF24G.

\section{APPENDIX A \\ SPITZER ASTRONOMICAL OBSERVATION REQUESTS (AORS) FOR UNPUBLISHED CLOUDS}

Three clouds listed in Table 1 do not have published data references (Chamaeleon I, Chamaeleon III, and Musca). Additionally, only a small piece of Aquila was published in the reference listed for that cloud (Gutermuth et al. 2008). Consequently, for these four clouds there are no published lists of the Spitzer AORs that targeted them. We thus list their AORs in Table 8.

\section{APPENDIX B ROUNDING ARTIFACTS IN COLOR-COLOR DIAGRAMS}

Inspection of Figure 2 reveals a vertical line of sources at [3.6]-[4.5] 0.5. If an object has identical flux densities at two Spitzer wavelengths, the magnitude difference between these two wavelengths is then simply equal to:

$$
m_{1}-m_{2}=2.5 \log \frac{f f z m_{2}}{f f z m_{1}}
$$

where $f f z m_{1}$ and $f f z m_{2}$ are the fluxes for zero magnitude at each wavelength. For 3.6 and $4.5 \mu \mathrm{m}$, this gives [3.6]-[4.5] $=0.473$.

There are 147 objects plotted in Figure 2 with identical flux densities at 3.6 and $4.5 \mu \mathrm{m}$, resulting in 147 objects plotted at the same value on the $x$-axis and thus giving rise to the vertical line of sources. We note that this is an artifact introducing by rounding all flux densities to two significant digits. To demonstrate this point, Figure 10 plots the same color-color diagram as Figure 2, except now using the original data before rounding. As evident from inspection of this figure, the vertical line of sources at [3.6]-[4.5] 0.5 has now disappeared.

The same artifact is also present in Figure 2 in the [3.6]-[24] color. For 3.6 and $24 \mu \mathrm{m}$, identical flux densities result in a magnitude difference of [3.6]-[24] $=3.959$. There are only 23 objects plotted in Figure 2 with identical flux densities at 3.6 and $24 \mu \mathrm{m}$, so while the vertical line of sources at [3.6]-[24] 4 is present, it is not as apparent. Similar artifacts also affect the colors plotted in Figures 8 and 9 using the extinction corrected photometry. For the four colors plotted in these two figures, between 38 and 95 objects with identical flux densities are plotted. While corresponding vertical and horizontal lines of sources are present, none are apparent because they all occur at colors where the source densities are too high for these small subsets of sources to be visible. These rounding artifacts have no effect on the results presented in this paper.

\section{APPENDIX C BOLOMETRIC TEMPERATURES OF CLASS II AND III SOURCES}

As noted in Section 3.1, the bolometric temperatures of our Class II and III YSOs are generally tightly clustered into two narrow $T_{\text {bol }}^{\prime}$ ranges (see Figures 4 and 7). To understand the origins of these effects, we construct simple continuum radiative transfer models of circumstellar disks using the three-dimensional Monte Carlo radiative transfer package RADMC-3D. ${ }^{25}$ We assume an axisymmetric physical structure and use RADMC-3D to first calculate the two-dimensional dust temperature profile through the disks and then generate full model SEDs from optical to millimeter wavelengths.

We adopt a simple, parameterized disk density structure following a power law in the radial coordinate and a Gaussian in the vertical coordinate. The exact density profile is given as follows:

$$
\rho_{\text {disk }}(s, z)=\rho_{0}\left(\frac{s}{s_{o}}\right)^{-\alpha} \exp \left[-\frac{1}{2}\left(\frac{z}{H_{s}}\right)^{2}\right]
$$

where $z$ is the distance above the midplane $(z=r \cos \theta$, with $r$ and $\theta$ the usual radial and zenith angle spherical coordinates), $s$ is the distance in the midplane from the origin $\left(s=\sqrt{r^{2}-z^{2}}\right)$, and $\rho_{0}$ is the density at the reference midplane distance $s_{0}$, normalized such that the total disk mass is correct (see below). The quantity $H_{s}$ is the disk scale height and is given by $H_{s}=H_{o}\left(\frac{s}{s_{o}}\right)^{\beta}$, where $H_{o}$ is the scale height at $s_{o}$. The parameter $\beta$, which describes how the scale height changes with $s$ and sets the flaring of the disk, is treated as a free parameter (see below). We set $H_{o}=10 \mathrm{AU}$ at $s_{o}=100 \mathrm{AU}$. The disk surface density profile, calculated by integrating Equation (5) over the vertical coordinate $z$, has a radial powerlaw index of $\Sigma(s) \propto s^{-p}$, where $p=\alpha-\beta$. For these models, we hold $\alpha$ fixed at 2.25 and the disk outer radius fixed at $100 \mathrm{AU}$.

\footnotetext{
25 Available at: http://www.ita.uni-heidelberg.de/ dullemond/software/ radmc-3d/
} 

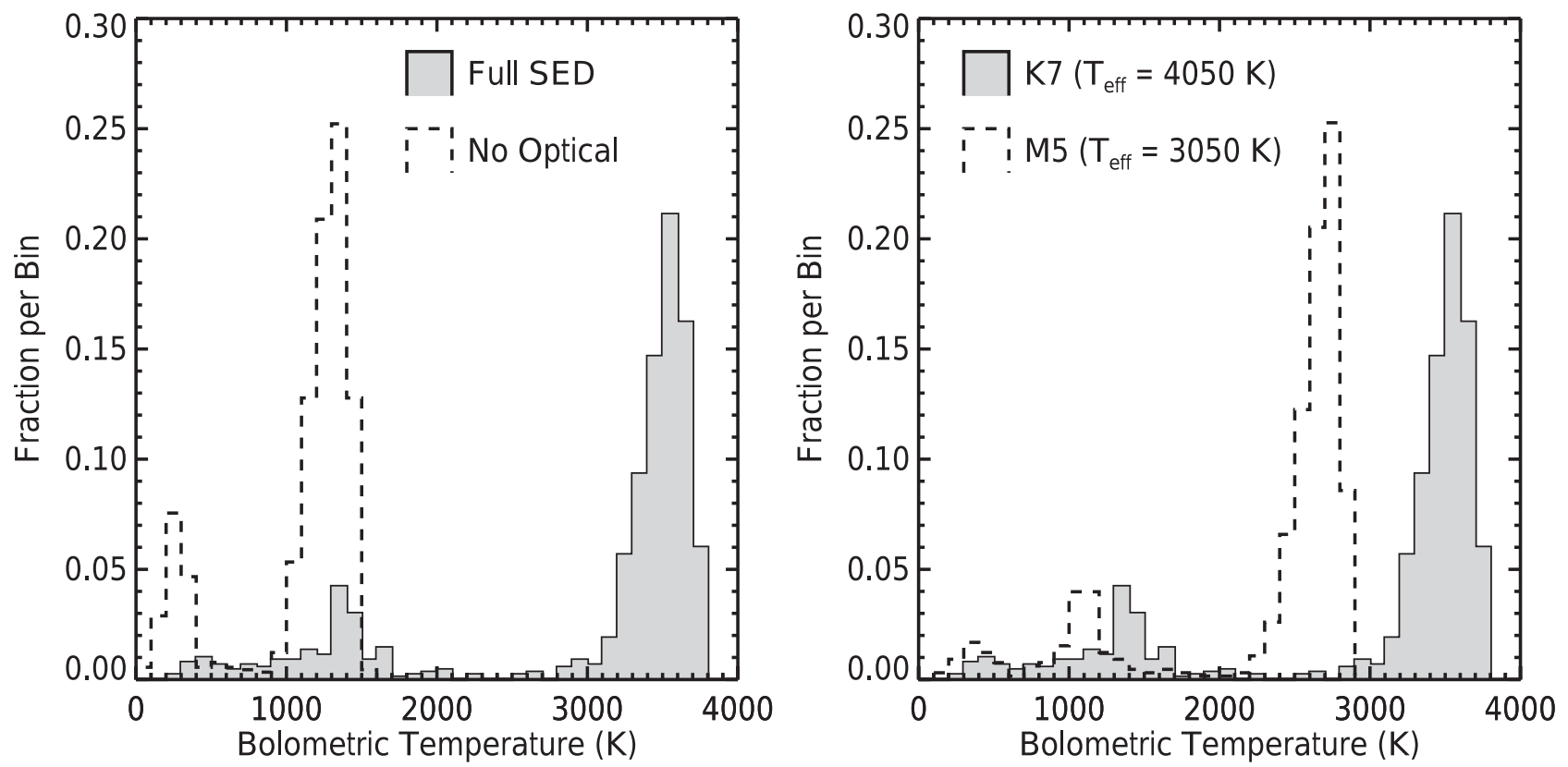

Figure 11. Histograms showing the distribution of $T_{\text {bol }}^{\prime}$ for the models described in the text. Left: the distributions of $T_{\text {bol }}^{\prime}$ for the K7 disk models, calculated both with (shaded) and without (dashed) optical photometry included in the SEDs. Right: the distributions of $T_{\text {bol }}^{\prime}$ for the K7 (shaded) and M5 (dashed) disk models, in both cases calculated with optical photometry included in the SEDs.

To consider a diverse set of disks, we treat the disk inner radius, flaring parameter $\beta$, and total disk mass as free parameters. Allowed values for each parameter are as follows: $0.1,1,10,20$, and $50 \mathrm{AU}$ for the disk inner radius; $1.0,1.25$, $1.5,1.75$ for $\beta$, and $0.0005,0.001,0.005,0.01$, and $0.05 M_{\odot}$ for the disk mass. We thus consider 100 total models and generate SEDs at 9 inclinations ${ }^{26}$ starting at $i=5^{\circ}$ and increasing in steps of $10^{\circ}$, resulting in 900 total model SEDs. We adopt the dust opacities of Ossenkopf \& Henning (1994) appropriate for thin ice mantles after $10^{5}$ year of coagulation at a gas density of $10^{6} \mathrm{~cm}^{-3}$ (OH5 dust). The radiative transfer is performed with no external heating by the interstellar radiation field, but isotropic scattering off dust grains is included. The input stellar spectrum is assumed to be a simple blackbody with $L=1 L_{\odot}$ and $T_{\text {eff }}$ of either 4050 or $3050 \mathrm{~K}$ (corresponding approximately to K7 and M5 spectral type pre-main sequence stars, respectively; Pecaut \& Mamajek 2013). Finally, for each of the 1800 total model SEDs (two stellar models each surrounded by 100 different circumstellar disk models, each viewed at 9 inclinations), we calculate two values of the bolometric temperature: one with the full SED from optical to millimeter wavelengths, and another excluding all optical wavelengths (all wavelengths shortward of $1.25 \mu \mathrm{m}$ ).

The left panel of Figure 11 shows the distributions of $T_{\mathrm{bol}}^{\prime}$ for the K7 disk models, calculated both with and without optical photometry included in the SEDs. Since the radiative transfer model includes no foreground extinction, these values are equivalent to our observed values after correcting for extinction. When optical photometry is included in the SEDs, the distribution of $T_{\text {bol }}^{\prime}$ exhibits a narrow peak around $3500 \mathrm{~K}$ with a width of only a few hundred $\mathrm{K}$, plus a long tail to lower values corresponding to SEDs viewed at edge-on inclinations. A similar behavior is exhibited when optical photometry is left out of the SEDs, except both the peak and long tail are shifted

\footnotetext{
${ }^{26}$ An inclination of $i=0^{\circ}$ corresponds to a pole-on (face-on) system, while an inclination of $i=90^{\circ}$ corresponds to an edge-on system.
}

to lower values of $T_{\mathrm{bol}}^{\prime}$. This behavior is expected since the inclusion of shorter wavelength data shifts the flux-weighted mean frequency to higher values.

The right panel of Figure 11 shows the distributions of $T_{\text {bol }}^{\prime}$ for the K7 and M5 disk models, in both cases calculated with optical photometry included in the SEDs. Each set of models exhibits narrow peaks, with the K7 model peak located at higher values than the M5 model. Even for the large range of disk properties considered here, it is evident from this figure that the measured $T_{\text {bol }}^{\prime}$ for YSOs with disks is mostly determined by the spectral type of the central source. The disk properties introduce only a very narrow spread except for models viewed at nearly edge-on inclinations.

The tight clustering into two narrow ranges of $T_{\mathrm{bol}}^{\prime}$ for most of our Class II and III YSOs, as evident from Figures 4 and 7, is naturally explained by these results. A K7 spectral type is assumed for most sources when deriving extinction corrections, explaining the tight clustering, and optical photometry is only available for a subset of our YSOs (see Section 2.4), explaining the bifurcation into two separate ranges of $T_{\mathrm{bol}}^{\prime}$. One consequence of this situation is that, even though a boundary in bolometric temperature between Class II and Class III is defined by Chen et al. (1995), $T_{\mathrm{bol}}^{\prime}$ is not a good discriminator between these two Classes.

\section{APPENDIX D \\ TABLES OF ANCILLARY PHOTOMETRY COLLECTED FROM THE LITERATURE}

Here we tabulate all ancillary photometry used to construct YSO SEDs, where we define ancillary to mean all photometry beyond the 2MASS and Spitzer photometry measured, bandmerged, and cataloged by the c2d pipeline. This definition of ancillary includes Spitzer $160 \mu$ m photometry. All flux densities and flux density uncertainties are listed in mJy and rounded to two significant digits. 
Tables 9 and 10 list, for each YSO, the same running index as in Table 2, followed by flux density and flux density uncertainty pairs for all photometry compiled between 0.36 and $0.96 \mu \mathrm{m}$. Table 9 lists observed values whereas Table 10 lists extinction corrected values.

Tables 11 and 12 list, for each YSO, the same running index as in Table 2, followed by flux density and flux density uncertainty pairs for all ancillary photometry compiled between 3.4 and $22 \mu \mathrm{m}$. Table 11 lists observed values whereas Table 12 lists extinction corrected values.

Tables 13 and 14 list, for each YSO, the same running index as in Table 2, followed by flux density and flux density uncertainty pairs for all ancillary photometry compiled between 3.4 and $22 \mu \mathrm{m}$. Table 13 lists observed values whereas Table 14 lists extinction corrected values.

\section{REFERENCES}

Adams, F. C., Lada, C. J., \& Shu, F. H. 1987, ApJ, 312, 788

Alcalá, J. M., Spezzi, L., Chapman, N., et al. 2008, ApJ, 676, 427

Alexander, R. D., \& Armitage, P. J. 2009, ApJ, 704, 989

Allen, L. E., Calvet, N., D'Alessio, P., et al. 2004, ApJS, 154, 363

André, P., Men'shchikov, A., Bontemps, S., et al. 2010, A\&A, 518, L102

Andre, P., Ward-Thompson, D., \& Barsony, M. 1993, ApJ, 406, 122

Bell, C. P. M., Naylor, T., Mayne, N. J., Jeffries, R. D., \& Littlefair, S. P. 2013, MNRAS, 434, 806

Belloche, A., Parise, B., Schuller, F., et al. 2011a, A\&A, 535, A2

Belloche, A., Schuller, F., Parise, B., et al. 2011b, A\&A, 527, A145

Bontemps, S., André, P., Kaas, A. A., et al. 2001, A\&A, 372, 173

Bressert, E., Bastian, N., Gutermuth, R., et al. 2010, MNRAS, 409, L54

Broekhoven-Fiene, H., Matthews, B. C., Harvey, P. M., et al. 2014, ApJ, 786, 37

Chapman, N. L., Lai, S.-P., Mundy, L. G., et al. 2007, ApJ, 667, 288

Chapman, N. L., Mundy, L. G., Lai, S.-P., \& Evans, N. J., II 2009, ApJ, 690, 496

Chen, H., Myers, P. C., Ladd, E. F., \& Wood, D. O. S. 1995, ApJ, 445, 377

Cieza, L. A., Schreiber, M. R., Romero, G. A., et al. 2010, ApJ, 712, 925

Cieza, L. A., Schreiber, M. R., Romero, G. A., et al. 2012, ApJ, 750, 157

Clube, S. V. M. 1967, MNRAS, 137, 189

Comerón, F. 2008, in Hand book of Star Forming Regions, Vol. II, The Southern Sky, ed. B. Reipurth (San Francisco, CA: ASP), 295

Comeron, F., Torra, J., \& Gomez, A. E. 1992, Ap\&SS, 187, 187

Crapsi, A., van Dishoeck, E. F., Hogerheijde, M. R., Pontoppidan, K. M., \& Dullemond, C. P. 2008, A\&A, 486, 245

D'Antona, F., \& Mazzitelli, I. 1994, ApJS, 90, 467

de Geus, E. J., de Zeeuw, P. T., \& Lub, J. 1989, A\&A, 216, 44

de Zeeuw, P. T., Hoogerwerf, R., de Bruijne, J. H. J., Brown, A. G. A., \& Blaauw, A. 1999, AJ, 117, 354

Di Francesco, J., Johnstone, D., Kirk, H., MacKenzie, T., \& Ledwosinska, E. 2008, ApJS, 175, 277

Dowell, C. D., Allen, C. A., Babu, R. S., et al. 2003, Proc. SPIE, 4855, 73

Dunham, M. M., Arce, H. G., Allen, L. E., et al. 2013, AJ, 145, 94

Dunham, M. M., Crapsi, A., Evans, N. J., II, et al. 2008, ApJS, 179, 249

Dunham, M. M., Evans, N. J., II, Bourke, T. L., et al. 2006, ApJ, 651, 945

Dunham, M. M., Evans, N. J., II, Terebey, S., Dullemond, C. P., \& Young, C. H. 2010, ApJ, 710, 470

Dunham, M. M., Stutz, A. M., Allen, L. E., et al. 2014, in Protostars and Planets VI, ed. H. Beuther et al. (Tuscon, AZ: Univ. Arizona Press), 195

Dzib, S., Loinard, L., Mioduszewski, A. J., et al. 2011, RMxAA, 40, 231

Enoch, M. L., Evans, N. J., II, Sargent, A. I., \& Glenn, J. 2009, ApJ, 692, 973

Enoch, M. L., Glenn, J., Evans, N. J., II, et al. 2007, ApJ, 666, 982

Enoch, M. L., Young, K. E., Glenn, J., et al. 2006, ApJ, 638, 293

Evans, N. J., II, Allen, L. E., Blake, G. A., et al. 2003, PASP, 115, 965

Evans, N. J., II, Dunham, M. M., Jørgensen, J. K., et al. 2009, ApJS, 181, 321

Evans, N. J., II, Harvey, P. M., Dunham, M. M., et al. 2007, Final Delivery of Data from the c2d Legacy Project: IRAC and MIPS

Evans, N. J., II, Heiderman, A., \& Vutisalchavakul, N. 2014, ApJ, 782, 114

Fazio, G. G., Hora, J. L., Allen, L. E., et al. 2004, ApJS, 154, 10

Gould, B. A. 1879, RNAO, 1, D1

Greene, T. P., Wilking, B. A., Andre, P., Young, E. T., \& Lada, C. J. 1994 , ApJ, 434, 614

Gutermuth, R. A. 2005, PhD thesis, Univ. Rochester

Gutermuth, R. A., Bourke, T. L., Allen, L. E., et al. 2008, ApJL, 673, L151
Gutermuth, R. A., Megeath, S. T., Myers, P. C., et al. 2009, ApJS, 184, 18 Harvey, P., Merín, B., Huard, T. L., et al. 2007a, ApJ, 663, 1149

Harvey, P. M., Chapman, N., Lai, S.-P., et al. 2006, ApJ, 644, 307

Harvey, P. M., Fallscheer, C., Ginsburg, A., et al. 2013, ApJ, 764, 133

Harvey, P. M., Huard, T. L., Jørgensen, J. K., et al. 2008, ApJ, 680, 495

Harvey, P. M., Rebull, L. M., Brooke, T., et al. 2007b, ApJ, 663, 1139

Hatchell, J., Terebey, S., Huard, T., et al. 2012, ApJ, 754, 104

Heiderman, A., \& Evans, N. J., II 2015, arXiv:1503.06810

Heiderman, A., Evans, N. J., II, Allen, L. E., Huard, T., \& Heyer, M. 2010, ApJ, 723, 1019

Herschel, Sir, J. F. W. 1847, Results of Astronomical Observations Made During the Years 1834, 5, 6, 7, 8, at the Cape of Good Hope; Being the Completion of a Telescopic Survey of the Whole Surface of the Visible Heavens, Commenced in 1825 (London: Smith, Elder and Co.)

Hsieh, T.-H., \& Lai, S.-P. 2013, ApJS, 205, 5

Jørgensen, J. K., Harvey, P. M., Evans, N. J., II, et al. 2006, ApJ, 645, 1246

Kaas, A. A., Olofsson, G., Bontemps, S., et al. 2004, A\&A, 421, 623

Kirk, J. M., Ward-Thompson, D., Di Francesco, J., et al. 2009, ApJS, 185, 198

Knude, J., \& Hog, E. 1998, A\&A, 338, 897

Kraus, A. L., Ireland, M. J., Hillenbrand, L. A., \& Martinache, F. 2012, ApJ, 745,19

Kryukova, E., Megeath, S. T., Gutermuth, R. A., et al. 2012, AJ, 144, 31

Lada, C. J., Lombardi, M., \& Alves, J. F. 2009, ApJ, 703, 52

Lada, C. J., \& Wilking, B. A. 1984, ApJ, 287, 610

Lonsdale, C. J., Smith, H. E., Rowan-Robinson, M., et al. 2003, PASP, 115,897

Maury, A. J., André, P., Men'shchikov, A., Könyves, V., \& Bontemps, S. 2011, A\&A, 535, A77

Megeath, S. T., Gutermuth, R., Muzerolle, J., et al. 2012, AJ, 144, 192

Merín, B., Jørgensen, J., Spezzi, L., et al. 2008, ApJS, 177, 551

Muzerolle, J., Megeath, S. T., Gutermuth, R. A., et al. 2004, ApJS, 154, 379

Myers, P. C., Adams, F. C., Chen, H., \& Schaff, E. 1998, ApJ, 492, 703

Myers, P. C., Fuller, G. A., Mathieu, R. D., et al. 1987, ApJ, 319, 340

Myers, P. C., \& Ladd, E. F. 1993, ApJL, 413, L47

Neuhäuser, R., \& Forbrich, J. 2008, in Hand book of Star Forming Regions, Vol. II, The Southern Sky, ed. B. Reipurth (San Francisco, CA: ASP), 735

Noriega-Crespo, A., Moro-Martin, A., Carey, S., et al. 2004, ApJS, 154, 402 Offner, S. S. R., \& McKee, C. F. 2011, ApJ, 736, 53

Oliveira, I., Merín, B., Pontoppidan, K. M., et al. 2009, ApJ, 691, 672

Oliveira, I., Pontoppidan, K. M., Merín, B., et al. 2010, ApJ, 714, 778

Ossenkopf, V., \& Henning, T. 1994, A\&A, 291, 943

Padgett, D. L., Rebull, L. M., Stapelfeldt, K. R., et al. 2008, ApJ, 672, 1013

Pattle, K., Ward-Thompson, D., Kirk, J. M., et al. 2015, MNRAS, 450, 1094 Pecaut, M. J., \& Mamajek, E. E. 2013, ApJS, 208, 9

Persi, P., Marenzi, A. R., Gómez, M., \& Olofsson, G. 2003, A\&A, 399, 995 Peterson, D. E., Caratti o Garatti, A., Bourke, T. L., et al. 2011, ApJS, 194, 43 Pöppel, W. G. L. 2001, in ASP Conf. Ser. 243, From Darkness to Light: Origin and Evolution of Young Stellar Clusters, ed. T. Montmerle \& P. André (San Francisco, CA: ASP), 667

Porras, A., Jørgensen, J. K., Allen, L. E., et al. 2007, ApJ, 656, 493

Rebull, L. M., Padgett, D. L., McCabe, C.-E., et al. 2010, ApJS, 186, 259

Rebull, L. M., Stapelfeldt, K. R., Evans, N. J., II, et al. 2007, ApJS, 171, 447

Ribas, Á, Bouy, H., \& Merín, B. 2015, arXiv:1502.00631

Ribas, Á, Merín, B., Bouy, H., \& Maud, L. T. 2014, A\&A, 561, A54

Rieke, G. H., Young, E. T., Engelbracht, C. W., et al. 2004, ApJS, 154, 25

Robitaille, T. P., Whitney, B. A., Indebetouw, R., Wood, K., \& Denzmore, P. 2006, ApJS, 167, 256

Romero, G. A., Schreiber, M. R., Cieza, L. A., et al. 2012, ApJ, 749, 79

Sadavoy, S. I., Di Francesco, J., André, P., et al. 2014, ApJL, 787, L18

Shu, F. H. 1977, ApJ, 214, 488

Shu, F. H., Adams, F. C., \& Lizano, S. 1987, ARA\&A, 25, 23

Skrutskie, M. F., Cutri, R. M., Stiening, R., et al. 2006, AJ, 131, 1163

Soderblom, D. R., Hillenbrand, L. A., Jeffries, R. D., Mamajek, E. E., \& Naylor, T. 2014, in Protostars and Planets VI, ed. H. Beuther et al. (Tuscon, AZ: Univ. Arizona Press), 219

Spezzi, L., Vernazza, P., Merín, B., et al. 2011, ApJ, 730, 65

Stothers, R., \& Frogel, J. A. 1974, AJ, 79, 456

Stutz, A. M., Tobin, J. J., Stanke, T., et al. 2013, ApJ, 767, 36

Suresh, A., Dunham, M. M., Arce, H. G., et al. 2015, AJ, submitted

Terebey, S., Shu, F. H., \& Cassen, P. 1984, ApJ, 286, 529

Tobin, J. J., Hartmann, L., Looney, L. W., \& Chiang, H.-F. 2010, ApJ, 712,1010

Ward-Thompson, D., di Francesco, J., Hatchell, J., et al. 2007, PASP, 119, 855 Weingartner, J. C., \& Draine, B. T. 2001, ApJ, 548, 296 
Werner, M. W., Roellig, T. L., Low, F. J., et al. 2004, ApJS, 154, 1

Whitney, B. A., Wood, K., Bjorkman, J. E., \& Wolff, M. J. 2003, ApJ, 591,1049

Whittet, D. C. B., Prusti, T., Franco, G. A. P., et al. 1997, A\&A, 327, 1194

Wilking, B. A., Gagné, M., \& Allen, L. E. 2008, Star Formation in the $\rho$ Ophiuchi Molecular Cloud, ed. B. Reipurth, 351

Wilking, B. A., Lada, C. J., \& Young, E. T. 1989, ApJ, 340, 823

Wright, E. L., Eisenhardt, P. R. M., Mainzer, A. K., et al. 2010, AJ, 140, 1868
Wu, J., Dunham, M. M., Evans, N. J., II, Bourke, T. L., \& Young, C. H. 2007, AJ, 133, 1560

Wyatt, M. C. 2008, ARA\&A, 46, 339

Young, C. H., \& Evans, N. J., II 2005, ApJ, 627, 293

Young, C. H., Jørgensen, J. K., Shirley, Y. L., et al. 2004, ApJS, 154, 396

Young, K. E., Enoch, M. L., Evans, N. J., II, et al. 2006, ApJ, 644, 326

Young, K. E., Harvey, P. M., Brooke, T. Y., et al. 2005, ApJ, 628, 283 